Article

\title{
Comparative Study of Some Numerical Methods for the Burgers-Huxley Equation
}

\author{
Appanah Rao Appadu ${ }^{1, *}$, Bilge İnan ${ }^{2}\left(\mathbb{D}\right.$ and Yusuf Olatunji Tijani ${ }^{1}(\mathbb{C}$ \\ 1 Department of Mathematics and Applied Mathematics, Nelson Mandela University, \\ Port-Elizabeth 6031, South Africa; s221356053@mandela.ac.za \\ 2 Department of Mathematics, Faculty of Muallim Rıfat Education, Kilis 7 Aralık University, \\ 79000 Kilis, Turkey; drbilgeinan@gmail.com \\ * Correspondence: Rao.Appadu@mandela.ac.za or Rao.Appadu31@gmail.com
}

Received: 19 September 2019; Accepted: 19 October 2019; Published: 24 October 2019

check for updates

\begin{abstract}
In this paper, we construct four numerical methods to solve the Burgers-Huxley equation with specified initial and boundary conditions. The four methods are two novel versions of nonstandard finite difference schemes (NSFD1 and NSFD2), explicit exponential finite difference method (EEFDM) and fully implicit exponential finite difference method (FIEFDM). These two classes of numerical methods are popular in the mathematical biology community and it is the first time that such a comparison is made between nonstandard and exponential finite difference schemes. Moreover, the use of both nonstandard and exponential finite difference schemes are very new for the Burgers-Huxley equations. We considered eleven different combination for the parameters controlling diffusion, advection and reaction, which give rise to four different regimes. We obtained stability region or condition for positivity. The performances of the four methods are analysed by computing absolute errors, relative errors, $L_{1}$ and $L_{\infty}$ errors and CPU time.
\end{abstract}

Keywords: Burgers-Huxley equation; nonstandard finite difference method; explicit exponential finite difference method; fully implicit exponential finite difference method; absolute error; relative error.

\section{Introduction}

Numerical and mathematical analysis are of significant importance for the solution and understanding of problems in science and engineering. Such problems are usually expressed using differential equations. Many numerical and mathematical methods use geometrical and analytical properties of mathematical and/or numerical problems.

Non-linearities exist in almost every branch of science and engineering ranging from biological systems, organic and inorganic chemistry, population dynamics, astrophysics, robotics, biomolecular engineering and zoology [1]. Non-linear partial differential equations (NLPDE) are the resulting equations for many physical phenomena, which in turn has motivated many researchers to study these alluring non-linear problems. Since mathematicians regard existence as one of the prime attributes of studies [2], the existence, uniqueness, symmetry and integrability have been studied for many evolution parabolic equations (see [3-5]). Unfortunately, exact solution rarely exists for many of these non-linear problems. However, there are some non-linear partial differential equations that become integrable after some symbolic transformation. In this case, the analytical solution becomes obtainable. In this regard, many powerful techniques have been introduced, amongst them are Lie group method [6], Jacobi elliptic functions method [7], function expansion method [8,9], homogeneous balance method [10], solitary wave ansatz method [11], and Hirota bilinear method [12], to mention a few. The Burgers-Huxley (BH) equation, which can be seen as an archetypal equation for describing 
the interaction among reaction mechanism, convection effects and diffusion transport, has been widely studied by researchers. Many techniques for obtaining solution to the Burgers-Huxley (BH) equation have been provided, one of them being from the work of Ismail et al. [13]. They provided a solution to the generalised Burgers-Huxley equation using the Adomian decomposition method (ADM). Batiha et al. [14], Biazar and Mohammadi [15], Yaghouti and Zabili [16], Molabahramia and Khani [17], Mittal and Jiwari [18], Javidi [19] and Tomasiello [20] obtained solutions to the Burgers-Huxley equation using variational iteration method (VIM), differential transform method (DTM), Laplace decomposition method, homotopy analysis method (HAM), differential quadrature method, spectral collocation and IDQ methods, respectively. Ray and Gupta [21,22] constructed a novel Haar wavelet collocation method and used it for the numerical solutions of the Burgers, Boussinesq-Burgers, Huxley and Burgers-Huxley equations. Discontinuity is the major drawback of the Haar wavelet method. The approximate analytical methods are plagued with many downsides, which include slow convergence at large $t$, high computer memory usage and difficulty in finding a closed form formula for the obtained series expression. It has been duly noted that the use of standard time integration techniques such as forward or backward Euler and Runge-Kutta methods to solve such differential models often lead to numerical instabilities and chaotic solution due to selection of discretisation parameters [23]. Kyrychko et al. [24] presented a traveling wave analysis for the extended-Burgers-Huxley equation. The existence of traveling wave solution was also validated for singularly perturbed Burgers-Huxley equation.

Among various techniques for solving partial differential equations especially in mathematical biology, the NSFD methods have been proved to be one of the most efficient approaches in recent years $[25,26]$ due to positive definiteness under some conditions, boundedness of solution, monotonicity of the solutions and properties such as special solutions with predetermined stability. Nonstandard (NSFD) methods have been used since 1994 and, in the early stages, the linear advection and advection-diffusion equations were discretised using this type of method. Mickens and Gumel [27] presented a detailed explanation on the nonstandard technique for the Burgers-Fisher equation and the paper was published in 2002. Zhang et al. [28] constructed a scheme based on exact solution and the nonstandard finite difference schemes for the Burgers and Burgers-Fisher equation; their paper was published in 2014. Recently, Agbavon and Appadu [29] analysed four nonstandard schemes for the FitzHugh-Nagumo equation. Interested readers can check the following references for works where nonstandard finite difference scheme are employed (Appadu et al. [30], Appadu [31], Agbavon et al. [32] Chapwanya et al. [33], Mickens [34], Jordan [35], and Aderogba and Chapwanya [36]. The construction of NSFD methods for the Burgers-Huxley equation is very recent. Zibaei et al. [37] presented the exact and nonstandard schemes for the Burgers-Huxley equation and their paper was published in 2016. It is noteworthy to mention that the application of NSFD schemes are not limited to areas of Mathematical Biology; see the works of Oluwaseye and Talitha [38], Dai [39] and Diaz et al. [40].

Another class of schemes known as exponential finite difference methods have been used to solve the Burgers type equations. Some of the good points of these methods to mathematical biologists are the distinctive features of computational efficiency, lesser computational time, ability to preserve physical properties of the differential equation [41,42]. The exponential scheme was originally developed by Bhattacharry [43] to solve the heat equation and the paper was published in 1985. Inan and Bahadir [44] employed implicit and fully implicit exponential finite difference method to solve Burgers equation and their paper was published in 2013. Bahadir [45] obtained a numerical solution for the small time Korteweg-de Vries (KdV) equation using the exponential scheme. Macías-Díaz constructed a modified exponential method that preserves structural properties of the solutions of the Burgers-Huxley equation [42]. More generally, recently in 2017, Burgers-Huxley equations were solved using an explicit exponential finite difference method constructed by İnan [46]. In addition, Burgers equation was solved with exponential method modified with Padé approximation by İnan and Macías-Díaz [47] and by İnan [48]. The quite good accuracy of the exponential finite difference schemes and the 
nature of some of these alluring non-linear problems has made these methods quite popular for the Burgers-type equations.

The paper is organised as follows. In Section 2, we have a short overview of the Burgers-Huxley equation. Section 3 describes the numerical experiment. In Sections 4 and 5, we construct two versions of non-standard finite difference schemes and two versions of exponential finite difference schemes and study some of their properties. The results are presented in Section 6. Section 7, which is the concluding part, highlights the salient features of this paper and possible future extension of the present study. MATLAB R2015a and Fortran computing platforms were used for simulations. A very short version of this paper has been accepted for publication as [49].

\section{The Burgers-Huxley Equation}

In recent time, partial differential equations containing nonlinear diffusion such as Equation (1) played an important role in non-linear physics, physiology and nerve propagation. The generalised Burgers-Huxley equation describes a wide class of physical non-linear phenomena in biology. It is well-known that the generalised Burgers-Huxley equation has a traveling wave solution with properties like boundedness, monotonicity and positivity (see [24,50,51]).

The generalised Burgers-Huxley equation is given by

$$
\frac{\partial u}{\partial t}=\frac{\partial^{2} u}{\partial x^{2}}-\alpha u^{\delta} \frac{\partial u}{\partial x}+\beta u\left(1-u^{\delta}\right)\left(u^{\delta}-\gamma\right) .
$$

In population dynamics, $u(x, t)$ represent the population density, $\gamma$ is the species carrying capacity, $\alpha$ stands for the speed of advection and $\beta$ is a parameter which describe nonlinear source.

When certain condition is imposed on the parameter, the generalised Burgers-Huxley equation is reduced to many parabolic evolution equations of physical insight. When $\delta=1$ and $\beta=0$, Equation (1) is reduced to the Burgers equation, which can be used to study sound waves in viscous medium [52]. The FitzHugh-Nagumo equation is obtained when $\delta=1$ and $\alpha=0$; this equation has enormous applications in neurophysiology, logistic population growth and auto catalytic reaction [29]. When $\delta=1, \alpha=0$ and $\gamma=-1$, we have the Newell-Whitehead-Segel equation [53]. For $\alpha=0$ and $\beta=0$, Equation (1) is reduced to the well-known heat conduction equation. When $\alpha=0$, the Huxley equation, which describes nerve pulse propagation, liquid crystal wall motion and nerve fiber, is obtained. We note that, for $\delta=1$, Equation (1) gives the Burgers-Huxley equation.

\section{Numerical Experiment}

We solve the Burgers-Huxley equation

$$
\begin{aligned}
\frac{\partial u}{\partial t}+\alpha u \frac{\partial u}{\partial x}-\frac{\partial^{2} u}{\partial x^{2}} & =\beta u(1-u)(u-\gamma) \\
& =\beta(1+\gamma) u^{2}-\beta \gamma u-\beta u^{3}
\end{aligned}
$$

subject to the following initial and boundary conditions [54]:

$$
u(x, 0)=\left[\frac{\gamma}{2}+\frac{\gamma}{2} \tanh \left(A_{1} x\right)\right], u(0, t)=\left[\frac{\gamma}{2}+\frac{\gamma}{2} \tanh \left(-A_{1} A_{2}\right) t\right], u(1, t)=\left[\frac{\gamma}{2}+\frac{\gamma}{2} \tanh \left(A_{1}\left(1-A_{2} t\right)\right)\right],
$$

where $\alpha>0, \beta>0,0<\gamma<1, x \in[0,1]$ and $t \in[0,10]$. The exact solution is given by [54]

$$
u(x, t)=\left[\frac{\gamma}{2}+\frac{\gamma}{2} \tanh \left(A_{1}\left(x-A_{2} t\right)\right)\right]
$$

where

$$
A_{1}=\frac{-\alpha+\sqrt{\alpha^{2}+8 \beta}}{8} \gamma \text { and } A_{2}=\frac{\alpha \gamma}{2}-\frac{(2-\gamma)\left(-\alpha+\sqrt{\alpha^{2}+8 \beta}\right)}{4}
$$


We note that the initial condition $u(x, 0)$ is non-negative i.e $u(x, 0) \geq 0$. In this work, we consider 11 different cases:

(1) $\alpha=0.5, \beta=0.5, \gamma=0.001$.

(2) $\alpha=0.5,(\beta>\alpha) \beta=2.0, \gamma=0.001$.

(3) $\alpha=0.5,(\beta>>\alpha) \beta=10.0, \gamma=0.001$. (singularly perturbed case)

(4) $\alpha>\beta(\alpha=2.0), \beta=0.5, \gamma=0.001$.

(5) $\alpha=0.5, \beta=0.5, \gamma=0.5$.

(6) $\alpha=0.5, \beta>\alpha(\beta=2.0), \gamma=0.5$.

(7) $\alpha=0.5,(\beta>>\alpha) \beta=10.0, \gamma=0.5$. (singularly perturbed case)

(8) $\alpha>\beta(\alpha=2.0), \beta=0.5, \gamma=0.5$.

(9) $\alpha=\beta=1.0, \gamma=0.001([13,14])$.

(10) $\alpha=0.5, \beta=0.5, \gamma=0.001$ and $k=0.1$ (FIEFDM)

(11) $\alpha=0.5, \beta=10.0, \gamma=0.5$ and $k=0.1$ (FIEFDM)

We chose the first eight cases to consider four different regimes, i.e when $\alpha=\beta, \beta>\alpha, \beta>>\alpha$ (singularly perturbed) and $\alpha>\beta$ and with two different values of $\gamma$, namely $\gamma=0.001$ and $\gamma=0.5$. We also considered Case 9 so that we can compare our results with other methods in $[13,14]$. The case $\beta>>\alpha$ is a very challenging one. In [32], the partial differential equation

$$
\frac{\partial u}{\partial t}-\frac{\partial^{2} u}{\partial x^{2}}=\beta u(1-u)
$$

was solved using $\beta=10^{4}$ and the initial condition consisted of exponential function. This is quite a challenging numerical experiment. Cases 10 and 11 were chosen to check the effectiveness of the fully implicit exponential finite difference method at other values of $k$.

We obtained numerical solutions for the numerical experiment using non-standard finite difference, and explicit and fully implicit exponential finite difference methods. The solution domains are discretised into cells as $\left(x_{j}, t_{n}\right)$ where $x_{j}=j h,(j=1,2, \ldots, N)$ and $t_{n}=n k,(n=1,2, \ldots)$, $h=\Delta x=\frac{1-0}{N-1}$ is the spatial mesh size and we choose $h=0.1$ for all computations in this paper. The temporal step size is $k=\Delta t$ and obtained by applying positivity condition or from stability analysis. $U_{j}^{n}$ represents numerical solution while $u(x, t)$ denotes the exact solution at point $\left(x_{j}, t_{n}\right)$.

\section{Nonstandard Finite Difference Scheme}

The pioneering work on non-standard finite difference scheme can be traced back to the works of Ronald Mickens [26,55-57]. The NSFD scheme is designed to resolve the issue of numerical instabilities and/or chaotic behaviour problems, which mostly plague many numerical methods. The concepts generally work on the principle of dynamical consistency which vary from one system to another. There are some major rules to be followed in the construction of such methods. They are listed below.

1. Non-local representation of linear and non-linear terms on the computational grid; E.g. $u_{n} \approx$ $2 U_{n}-U_{n+1}, u_{n}^{2} \approx\left(\frac{U_{n+1}+2 U_{n}+U_{n-1}}{4}\right) U_{n}, u_{n}^{3} \approx 2 U_{n}^{3}-U_{n}^{2} U_{n+1}$ etc.

2. Use of numerator and denominator functions $\psi(h)$ and $\phi(k)$, respectively with the property

$$
\lim _{h \rightarrow 0} \psi(h)=h, \text { and } \lim _{k \rightarrow 0} \phi(k)=k,
$$

where $\psi(h)=h+O\left(h^{2}\right)$ and $\phi(k)=k+O(k)^{2}$.

3. The difference equation should have the same order as the original differential equation. In general, when the order of the difference equation is larger than the order of the differential equation, spurious solutions will appear [58]. 
4. The discrete approximation should preserve some important properties of the corresponding differential equation. Properties such as boundedness and positivity should be preserved.

The scheme constructed by Zibaei et al. [37] for Equation (2) is given by

$$
\begin{array}{r}
\frac{U_{j}^{n+1}-U_{j}^{n}}{\Phi}=\left[\frac{U_{j+1}^{n}-2 U_{j}^{n}+U_{j-1}^{n}}{\Psi}\right]-\alpha U_{j}^{n+1} \frac{U_{j}^{n}-U_{j-1}^{n}}{\Gamma}+\beta(1+\gamma)\left(U_{j-1}^{n}\right)^{2}-\beta \gamma U_{j}^{n+1} \\
+\beta\left(\frac{1}{2}\left(U_{j-1}^{n}\right)^{3}-\frac{3}{2}\left(U_{j-1}^{n}\right)^{2} U_{j}^{n+1}\right),
\end{array}
$$

where

$$
\Phi=\frac{1-e^{-2 A_{1} A_{2} k}}{2 A_{1} A_{2}}, \Gamma=\frac{e^{2 A_{1} h}-1}{2 A_{1}}, \Psi=\Gamma^{2} .
$$

$A_{1}$ is described in Equation (5) and $A_{2}=\alpha+\frac{4-2 \gamma}{\gamma} A_{1}$.

We propose two versions of NSFD scheme as NSFD1 and NSFD2.

\subsection{NSFD1 Scheme}

Following the rules above, we construct NSFD1 scheme for Equation (2) as

$$
\begin{array}{r}
\frac{U_{j}^{n+1}-U_{j}^{n}}{\phi(k)}=\left[\frac{U_{j+1}^{n}-2 U_{j}^{n}+U_{j-1}^{n}}{[\psi(h)]^{2}}\right]-\alpha U_{j}^{n+1} \frac{U_{j}^{n}-U_{j-1}^{n}}{\psi(h)}+\beta(1+\gamma)\left[2\left(U_{j}^{n}\right)^{2}-U_{j}^{n} U_{j}^{n+1}\right] \\
-\beta \gamma U_{j}^{n+1}-\beta U_{j}^{n+1}\left(U_{j}^{n}\right)^{2} .
\end{array}
$$

The denominator functions are defined as

$$
\phi(k)=\frac{e^{\beta k}-1}{\beta}, \psi(h)=\frac{e^{\beta h}-1}{\beta} .
$$

We have approximated the linear and non-linear terms as follows:

$$
u^{3}\left(x_{j}, t_{n}\right) \approx U_{j}^{n+1}\left(U_{j}^{n}\right)^{2}, u^{2}\left(x_{j}, t_{n}\right) \approx 2\left(U_{j}^{n}\right)^{2}-U_{j}^{n} U_{j}^{n+1}, u\left(x_{j}, t_{n}\right) \approx U_{j}^{n+1} .
$$

Equation (8) above is an explicit scheme. By making $U_{j}^{n+1}$ the subject and noting that $R=\frac{\phi(k)}{[\psi(h)]^{2}}$ and $r=\frac{\phi(k)}{\psi(h)}$, we have

$$
U_{j}^{n+1}=\frac{(1-2 R) U_{j}^{n}+R\left(U_{j+1}^{n}+U_{j-1}^{n}\right)+2 \phi(k) \beta(1+\gamma)\left(U_{j}^{n}\right)^{2}}{1+\alpha r\left(U_{j}^{n}-U_{j-1}^{n}\right)+\phi(k) \beta \gamma+\Phi(k) \beta(1+\gamma) U_{j}^{n}+\phi(k) \beta\left(U_{j}^{n}\right)^{2}} .
$$

We then proceed to check positivity and boundedness of the scheme given by Equation (10).

Theorem 1 (Dynamical Consistency). If $1-2 R \geq 0$, the numerical solution from NSFD1 satisfies

$$
0 \leq U_{j}^{n} \leq \gamma, \Longrightarrow 0 \leq U_{j}^{n+1} \leq \gamma,
$$

for all considered values of $n$ and $j$. 
Proof. For positivity, we require $1-2 R \geq 0$. Substituting the appropriate form for $R$, we obtain

$$
\left(\frac{e^{\beta k}-1}{\beta}\right)\left(\frac{\beta}{e^{\beta h}-1}\right)^{2} \leq \frac{1}{2}
$$

which gives

$$
k \leq \frac{1}{\beta} \ln \left(1+\frac{\left(e^{\beta h}-1\right)^{2}}{2 \beta}\right) .
$$

On substituting $h=0.1$ and evaluating for some different values of $\beta$, we obtain

(a) $k \leq 5.251 \times 10^{-3}$ for $\beta=0.5$.

(b) $k \leq 5.515 \times 10^{-3}$ for $\beta=1.0$.

(c) $k \leq 6.090 \times 10^{-3}$ for $\beta=2.0$.

(d) $k \leq 1.377 \times 10^{-2}$ for $\beta=10.0$.

For boundedness of NSFD1, we assume $0 \leq U_{j}^{n} \leq \gamma$ for all considered values of $n$ and $j$. Therefore,

$$
\begin{array}{r}
\left(U_{j}^{n+1}-\gamma\right)\left(1+\alpha r\left(U_{j}^{n}-U_{j-1}^{n}\right)+\phi(k) \beta \gamma+\phi(k) \beta(1+\gamma) U_{j}^{n}+\phi(k) \beta\left(U_{j}^{n}\right)^{2}\right)=(1-2 R) U_{j}^{n}+ \\
R\left(U_{j+1}^{n}+U_{j-1}^{n}\right)+2 \phi(k) \beta(1+\gamma)\left(U_{j}^{n}\right)^{2}-\gamma-\alpha r \gamma\left(U_{j}^{n}-U_{j-1}^{n}\right)-\phi(k) \beta \gamma^{2}-\phi(k) \beta \gamma(1+\gamma) U_{j}^{n} \\
-\phi(k) \beta \gamma\left(U_{j}^{n}\right)^{2} \leq 2 \beta \phi(k) \beta\left(U_{j}^{n}\right)^{2}+2 \phi(k) \beta \gamma\left(U_{j}^{n}\right)^{2}-\alpha r\left(U_{j}^{n}-U_{j-1}^{n}\right) \\
-\phi(k) \beta \gamma^{2}-\phi(k) \beta \gamma\left(U_{j}^{n}\right)-\phi(k) \beta \gamma^{2} U_{j}^{n}-\phi(k) \beta \gamma\left(U_{j}^{n}\right)^{2} \\
=\phi(k) \beta \gamma\left(U_{j}^{n}\right)^{2}+\phi(k) \beta\left(2\left(U_{j}^{n}\right)^{2}-\gamma^{2}-\gamma U_{j}^{n}\right)-\alpha r \gamma\left(U_{j}^{n}-U_{j-1}^{n}\right)-\phi(k) \beta \gamma^{2} U_{j}^{n} \\
\leq \beta \phi(k) \gamma U_{j}^{n}\left(U_{j}^{n}-\gamma\right)-\alpha r \gamma\left(U_{j}^{n}-U_{j-1}^{n}\right) \leq 0 .
\end{array}
$$

Using Equation (13), we have $U_{j}^{n+1} \leq \gamma$. Thus, the NSFD1 scheme is bounded, provided the values from the initial conditions are non-negative and bounded.

\subsection{NSFD2 Scheme}

Using other non-local representations for the linear and non-linear terms as

$$
u^{3}\left(x_{j}, t_{n}\right) \approx 2 U_{j}^{n+1}\left(U_{j}^{n}\right)^{2}-\left(U_{j}^{n}\right)^{3}, u\left(x_{j}, t_{n}\right) \approx U_{j}^{n+1},
$$

we propose the following scheme to discretise Equation (2):

$$
\begin{aligned}
\frac{U_{j}^{n+1}-U_{j}^{n}}{\phi(k)}=\left[\frac{U_{j+1}^{n}-2 U_{j}^{n}+U_{j-1}^{n}}{[\psi(h)]^{2}}\right]-\alpha U_{j}^{n+1} \frac{U_{j}^{n}-U_{j-1}^{n}}{\psi(h)}+ & \beta(1+\gamma)\left(U_{j}^{n}\right)^{2}-\beta \gamma U_{j}^{n+1} \\
& -2 \beta U_{j}^{n+1}\left(U_{j}^{n}\right)^{2}+\beta\left(U_{j}^{n}\right)^{3} .
\end{aligned}
$$

By making $U_{j}^{n+1}$ the subject of the equation, we obtain

$$
U_{j}^{n+1}=\frac{(1-2 R) U_{j}^{n}+R\left(U_{j+1}^{n}+U_{j-1}^{n}\right)+\beta \phi(k)\left[(1+\gamma)\left(U_{j}^{n}\right)^{2}+\left(U_{j}^{n}\right)^{3}\right]}{1+\alpha r\left(U_{j}^{n}-U_{j-1}^{n}\right)+\phi(k) \beta \gamma+2 \phi(k) \beta\left(U_{j}^{n}\right)^{2}},
$$

We then proceed to check positivity and boundedness of NSFD2 scheme.

Theorem 2 (Dynamical Consistency). If $1-2 R \geq 0$, the numerical solution from NSFD2 satisfies

$$
0 \leq U_{j}^{n} \leq \gamma, \Longrightarrow 0 \leq U_{j}^{n+1} \leq \gamma,
$$


for all relevant values of $n$ and $j$.

Proof. For positivity, we require $1-2 R \geq 0$. We have the same condition as for NSFD1 scheme. For boundedness, we note that $0 \leq U_{j}^{n} \leq \gamma$, for all values of $n$ and $j$. We have

$$
\begin{array}{r}
\left(U_{j}^{n+1}-\gamma\right)\left(1+\alpha r\left(U_{j}^{n}-U_{j-1}^{n}\right)+\phi(k) \beta \gamma+2 \phi(k) \beta\left(U_{j}^{n}\right)^{2}\right)=(1-2 R) U_{j}^{n}+R\left(U_{j+1}^{n}+U_{j-1}^{n}\right) \\
+\beta \phi(k)\left((1+\gamma)\left(U_{j}^{n}\right)^{2}+\left(U_{j}^{n}\right)^{3}\right)-\gamma-\gamma r \alpha\left(U_{j}^{n}-U_{j-1}^{n}\right)-\gamma^{2} \phi(k) \beta-2 \phi(k) \beta \gamma\left(U_{j}^{n}\right)^{2} \\
=\beta \phi(k)\left(U_{j}^{n}\right)^{2}+\beta \phi(k) \gamma\left(U_{j}^{n}\right)^{2}+\beta \phi(k)\left(U_{j}^{n}\right)^{3}-\alpha r \gamma\left(U_{j}^{n}-U_{j-1}^{n}\right)-\beta \gamma^{2} \phi(k)-2 \phi(k) \beta \gamma\left(U_{j}^{n}\right)^{2} \\
=\beta \phi(k)\left(U_{j}^{n}\right)^{2}+\beta \phi(k)\left(U_{j}^{n}\right)^{3}-\alpha r \gamma\left(U_{j}^{n}-U_{j-1}^{n}\right)-\beta \gamma^{2} \phi(k)-\phi(k) \beta \gamma\left(U_{j}^{n}\right)^{2} \\
=\beta \phi(k)\left(U_{j}^{n}\right)^{2}\left(U_{j}^{n}-\gamma\right)-\alpha r \gamma\left(U_{j}^{n}-U_{j-1}^{n}\right)+\beta \phi(k)\left(\left(U_{j}^{n}\right)^{2}-\gamma^{2}\right) \\
=\left(\beta \phi(k)\left(U_{j}^{n}\right)^{2}+\beta \phi(k)\left(U_{j}^{n}+\gamma\right)\right)\left(U_{j}^{n}-\gamma\right)-\alpha r \gamma\left(U_{j}^{n}-U_{j-1}^{n}\right) \leq 0 .
\end{array}
$$

Hence, $U_{j}^{n+1} \leq \gamma$. Hence, we conclude that NSFD2 scheme is bounded. The positivity conditions guarantee non-negativity of the solution.

\section{Exponential Finite Difference Methods}

In the construction of exponential finite difference schemes, we use the following difference operators:

$$
\begin{aligned}
\delta_{t} U_{j}^{n} & =\frac{U_{j}^{n+1}-U_{j}^{n}}{k}, \\
\delta_{x}^{(1)} U_{j}^{n} & =\frac{U_{j+1}^{n}-U_{j-1}^{n}}{2 h}, \\
\delta_{x}^{(2)} U_{j}^{n} & =\frac{U_{j+1}^{n}-2 U_{j}^{n}+U_{j-1}^{n}}{h^{2}}, \\
\delta_{x}^{(1)} U_{j}^{n+1} & =\frac{U_{j+1}^{n+1}-U_{j-1}^{n+1}}{2 h}, \\
\delta_{x}^{(2)} U_{j}^{n+1} & =\frac{U_{j+1}^{n+1}-2 U_{j}^{n+1}+U_{j-1}^{n+1}}{h^{2}},
\end{aligned}
$$

for each $j \in\{1,2, \ldots, N\}$ and $n \in\{1,2, \ldots\}$. In addition, we introduce the new discrete operator

$$
\Lambda U_{j}^{n}=\frac{\ln \left(U_{j}^{n+1}\right)-\ln \left(U_{j}^{n}\right)}{k}=\frac{1}{k} \ln \left(\frac{U_{j}^{n+1}}{U_{j}^{n}}\right) .
$$

\subsection{Explicit Exponential Finite Difference Method (EEFDM)}

An explicit exponential finite difference method was proposed for the generalised Burgers-Huxley equation and numerical solutions for $\delta=1$ were presented by İnan in [46]. When Equation (2) is rearranged, the following equation is obtained

$$
\frac{\partial u}{\partial t}=\beta u(1-u)(u-\gamma)-\alpha u \frac{\partial u}{\partial x}+\frac{\partial^{2} u}{\partial x^{2}}
$$


Dividing by $u$, we obtain

$$
\frac{\partial \ln u}{\partial t}=\frac{1}{u}\left(\beta u(1-u)(u-\gamma)-\alpha u \frac{\partial u}{\partial x}+\frac{\partial^{2} u}{\partial x^{2}}\right)
$$

and using finite difference approximations for derivatives, we obtain

$$
\Lambda U_{j}^{n}=\frac{1}{U_{j}^{n}}\left[\beta U_{j}^{n}\left(1-U_{j}^{n}\right)\left(U_{j}^{n}-\gamma\right)-\alpha U_{j}^{n} \delta_{x}^{(1)} U_{j}^{n}+\delta_{x}^{(2)} U_{j}^{n}\right]
$$

Finally, using Equations (18), (19) and (22), a single expression for EEFDM scheme is

$$
\begin{aligned}
U_{j}^{n+1}= & U_{j}^{n} \exp \left\{\frac { k } { U _ { j } ^ { n } } \left[\beta U_{j}^{n}\left(1-U_{j}^{n}\right)\left(U_{j}^{n}-\gamma\right)\right.\right. \\
& \left.\left.-\alpha U_{j}^{n}\left(\frac{U_{j+1}^{n}-U_{j-1}^{n}}{2 h}\right)+\left(\frac{U_{j+1}^{n}-2 U_{j}^{n}+U_{j-1}^{n}}{h^{2}}\right)\right]\right\} .
\end{aligned}
$$

To obtain the stability of EEFDM, we consider the corresponding standard finite difference scheme given by

$$
\frac{U_{j}^{n+1}-U_{j}^{n}}{k}=\left(\frac{U_{j+1}^{n}-2 U_{j}^{n}+U_{j-1}^{n}}{h^{2}}\right)-\alpha U_{j}^{n} \frac{U_{j+1}^{n}-U_{j-1}^{n}}{2 h}+\beta(1+\gamma)\left(U_{j}^{n}\right)^{2}-\beta \gamma U_{j}^{n}-\beta\left(U_{j}^{n}\right)^{3} .
$$

which can be rewritten in the form

$$
U_{j}^{n+1}=U_{j}^{n}+k\left(\frac{U_{j+1}^{n}-2 U_{j}^{n}+U_{j-1}^{n}}{h^{2}}\right)-k \alpha U_{j}^{n} \frac{U_{j+1}^{n}-U_{j-1}^{n}}{2 h}+k \beta(1+\gamma)\left(U_{j}^{n}\right)^{2}-k \beta \gamma U_{j}^{n}-k \beta\left(U_{j}^{n}\right)^{3} .
$$

We follow the idea of Taha and Ablowitz [59] by using the freezing coefficients method and Von Neumann stability analysis. We obtain the amplification factor as:

$$
\xi=1-I \frac{k \alpha}{h} U_{\max } \sin w+\frac{k}{h^{2}}(2 \cos w-2)+k \beta U_{\max }(1+\gamma)-k \beta \gamma-k \beta U_{\max }^{2} .
$$

Since $0 \leq U \leq \gamma$, it follows that $U_{\max }=\gamma$. On simplification, we obtain

$$
|\xi|=\sqrt{\left(1-\frac{4 k}{h^{2}} \sin ^{2} \frac{w}{2}\right)^{2}+\left(\frac{k \alpha \gamma}{h} \sin w\right)^{2}}
$$

Stability is guaranteed when $0<|\xi| \leq 1$ for $w=[-\pi, \pi]$. Table 1 shows as follow:

Table 1. Range of values of $k$ for stability of EEFDM with $h=0.1$.

\begin{tabular}{ccc}
\hline Cases & Parameter Values & Condition for Stability \\
\hline 1 & $\alpha=0.5, \beta=0.5, \gamma=0.001$ & $k \leq 0.005$ \\
2 & $\alpha=0.5, \beta=2.0, \gamma=0.001$ & $k \leq 0.005$ \\
3 & $\alpha=0.5, \beta=10.0, \gamma=0.001$ & $k \leq 0.005$ \\
4 & $\alpha=2.0, \beta=0.5, \gamma=0.001$ & $k \leq 0.005$ \\
5 & $\alpha=0.5, \beta=0.5, \gamma=0.5$ & $k \leq 0.005$ \\
6 & $\alpha=0.5, \beta=2.0, \gamma=0.5$ & $k \leq 0.005$ \\
7 & $\alpha=0.5, \beta=10.0, \gamma=0.5$ & $k \leq 0.005$ \\
8 & $\alpha=2.0, \beta=0.5, \gamma=0.5$ & $k \leq 0.005$ \\
9 & $\alpha=1.0, \beta=1.0, \gamma=0.001$ & $k \leq 0.005$ \\
\hline
\end{tabular}

We fix $h=0.1$ and we obtain the 3D plots of $|\xi|$ vs. $k$ vs. $\omega \in[-\pi, \pi]$. As shown in Figures 1-9. 


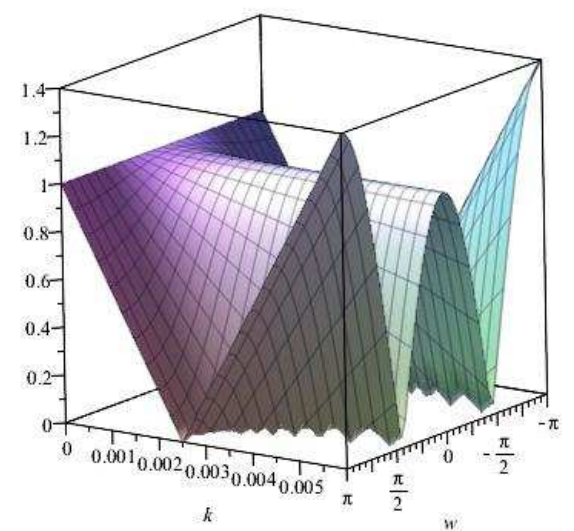

Figure 1. Plot of $|\xi|$ vs. $k$ vs. $\omega$ for $\alpha=0.5, \beta=0.5, \gamma=0.001$.

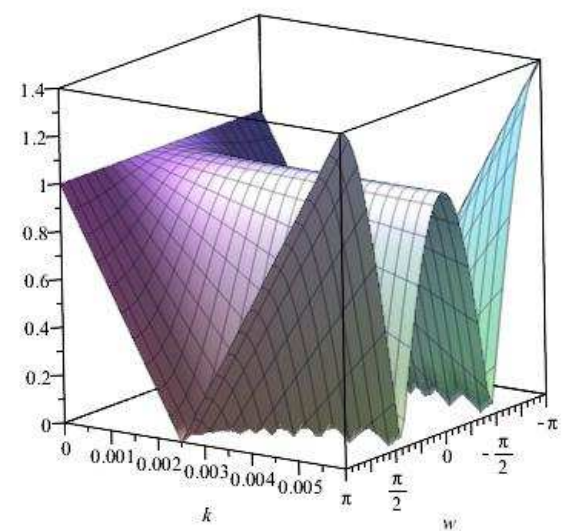

Figure 2. Plot of $|\xi|$ vs. $k$ vs. $\omega$ for $\alpha=0.5, \beta=2.0, \gamma=0.001$.

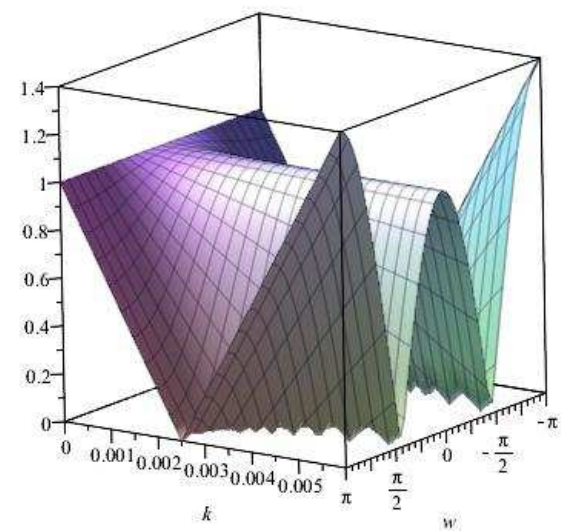

Figure 3. Plot of $|\xi|$ vs. $k$ vs. $\omega$ for $\alpha=0.5, \beta=10.0, \gamma=0.001$. 


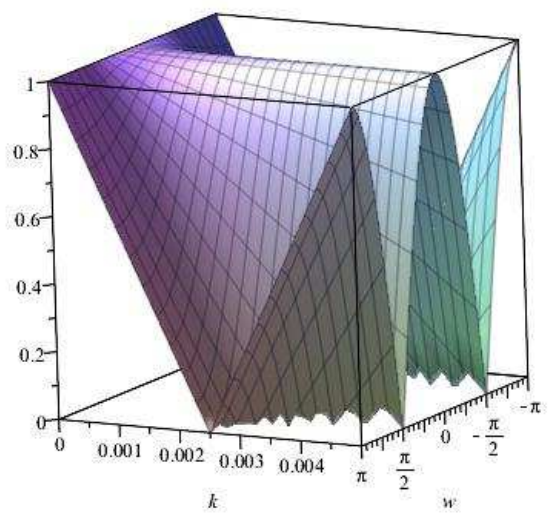

Figure 4. Plot of $|\xi|$ vs. $k$ vs. $\omega$ for $\alpha=2.0, \beta=0.5, \gamma=0.001$.

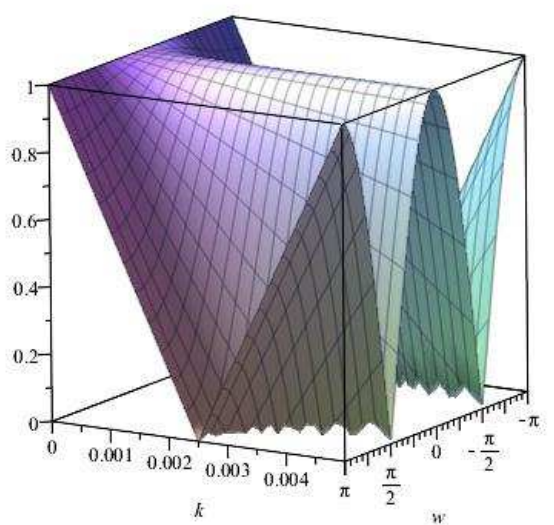

Figure 5. Plot of $|\xi|$ vs. $k$ vs. $\omega$ for $\alpha=0.5, \beta=0.5, \gamma=0.5$.

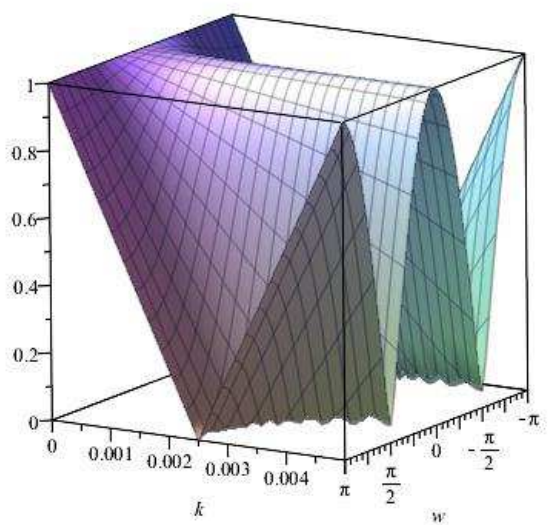

Figure 6. Plot of $|\xi|$ vs. $k$ vs. $\omega$ for $\alpha=0.5, \beta=2.0, \gamma=0.5$. 


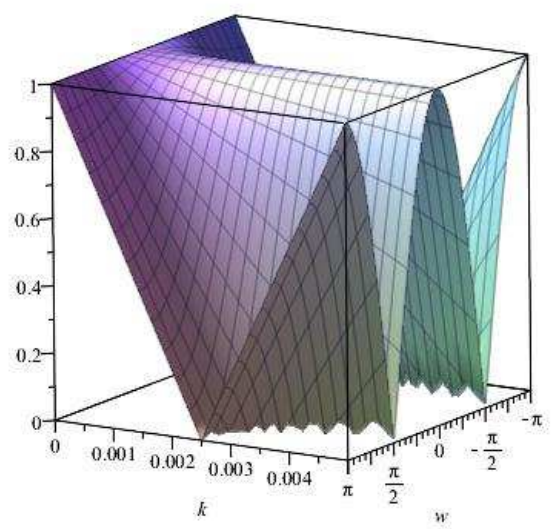

Figure 7. Plot of $|\xi|$ vs. $k$ vs. $\omega$ for $\alpha=0.5, \beta=10.0, \gamma=0.5$.

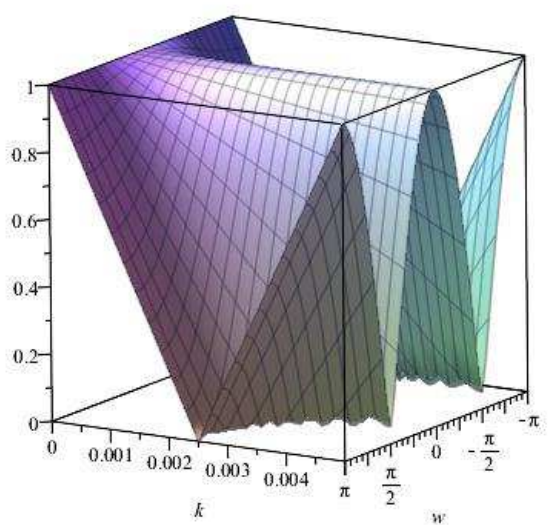

Figure 8. Plot of $|\xi|$ vs. $k$ vs. $\omega$ for $\alpha=2.0, \beta=0.5, \gamma=0.5$.

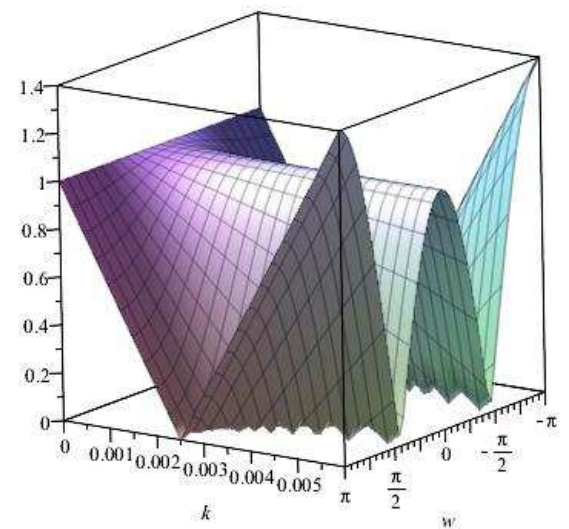

Figure 9. Plot of $|\xi|$ vs. $k$ vs. $\omega$ for $\alpha=1.0, \beta=1.0, \gamma=0.001$.

5.2. Fully Implicit Exponential Finite Difference Method (FIEFDM)

We rearrange Equation (2) to obtain

$$
\frac{\partial u}{\partial t}=\frac{\partial^{2} u}{\partial x^{2}}-\alpha u \frac{\partial u}{\partial x}+\beta u(1-u)(u-\gamma)
$$


Dividing by $u$ gives

$$
\frac{\partial \ln u}{\partial t}=\frac{1}{u}\left(\frac{\partial^{2} u}{\partial x^{2}}-\alpha u \frac{\partial u}{\partial x}+\beta u(1-u)(u-\gamma)\right),
$$

and using finite difference approximations for derivatives, we obtain following equation.

$$
\Lambda U_{j}^{n}=\frac{1}{U_{j}^{n}}\left[\beta U_{j}^{n+1}\left(1-U_{j}^{n+1}\right)\left(U_{j}^{n+1}-\gamma\right)-\alpha U_{j}^{n+1} \delta_{x}^{(1)} U_{j}^{n+1}+\delta_{x}^{(2)} U_{j}^{n+1}\right] .
$$

Finally, using Equations (20)-(33) gives

$$
\begin{array}{r}
U_{j}^{n+1}=U_{j}^{n} \exp \left(\frac { k } { U _ { j } ^ { n } } \left[\left(\frac{U_{j+1}^{n+1}-2 U_{j}^{n+1}+U_{j-1}^{n+1}}{h^{2}}\right)-\alpha U_{j}^{n+1}\left(\frac{U_{j+1}^{n+1}-U_{j-1}^{n+1}}{2 h}\right)+\right.\right. \\
\left.\left.\beta U_{j}^{n+1}\left(1-U_{j}^{n+1}\right)\left(U_{j}^{n+1}-\gamma\right)\right]\right),
\end{array}
$$

which is valid for values of $j$ lying in the interval $1 \leq j \leq N-1$. Equation (34) is a system of nonlinear difference equations. Let us consider these nonlinear systems of equations in the form

$$
\mathbf{F}(\mathbf{V})=\mathbf{0},
$$

where $\mathbf{F}=\left[f_{1}, f_{2}, \ldots, f_{N-1}\right]^{T}$ and $\mathbf{V}=\left[U_{1}^{n+1}, U_{2}^{n+1}, \ldots, U_{N-1}^{n+1}\right]^{T}$.

Newton's method applied to Equation (35) results in the following iteration:

1. Set $\mathbf{V}^{(0)}$, an initial guess.

2. For $m=0,1,2, .$. until convergence do:

Solve $J\left(\mathbf{V}^{(m)}\right) \boldsymbol{\Omega}^{(m)}=-\mathbf{F}\left(\mathbf{V}^{(m)}\right)$;

Set $\mathbf{V}^{(m+1)}=\mathbf{V}^{(m)}+\mathbf{\Omega}^{(m)}$ where $J\left(\mathbf{V}^{(m)}\right)$ is the Jacobian matrix which is evaluated analytically. The solution at the previous time-step is taken as the initial estimate. The Newton's iteration at each time-step is stopped when

$$
\left\|\mathbf{F}\left(\mathbf{V}^{(m)}\right)\right\|_{\infty} \leq 10^{-5} .
$$

We choose $10^{-5}$ as tolerance. It is not easy to choose a tolerance less than $10^{-5}$ due to excessive computational time.

\section{Numerical Results}

We performed the numerical experiment described in Section 3 to check the effectiveness of the two NSFD schemes and two exponential schemes. The accuracy of the proposed methods was measured using the absolute error, relative error, $L_{1}$ and $L_{\infty}$ errors.

$$
\begin{gathered}
\text { Absolute Error }=\left|u\left(x_{j}, t_{n}\right)-U\left(x_{j}, t_{n}\right)\right|, \\
\text { Relative Error }=\frac{\left|u\left(x_{j}, t_{n}\right)-U\left(x_{j}, t_{n}\right)\right|}{\left|u\left(x_{j}, t_{n}\right)\right|}, \\
L_{1}=h \sum_{j=1}^{N}\left|u\left(x_{j}, t_{n}\right)-U\left(x_{j}, t_{n}\right)\right|,
\end{gathered}
$$

and

$$
L_{\infty}=\max \left|u\left(x_{j}, t_{n}\right)-U\left(x_{j}, t_{n}\right)\right|
$$


All simulations were performed with $h=0.1$; the value of $k=0.005$ was carefully chosen to preserve the positivity and boundedness condition and/or the stability condition for the two NSFD schemes and two exponential finite difference methods. Comparison of the numerical solutions, exact solution, absolute error, relative error, $L_{1}$ and $L_{\infty}$ errors, CPU time for various values of $\alpha, \beta$ and $\gamma$ are made in Tables $2-38$ and in Figures 10-29. We also present results using implicit exponential finite difference scheme using $\alpha=0.5, \beta=0.5, \gamma=0.001$ and $\alpha=0.5, \beta=10.0, \gamma=0.5$ using temporal step size $k=0.1$ and spatial step size $h=0.1$.

In Case 1, parameters are chosen as $\alpha=\beta=0.5$ and $\gamma=0.001$. Tables 2-5 display the results for Case 1 at $t=1.0$ and $t=10.0$.

In Case 2, parameters are chosen as $\alpha=0.5, \beta=2.0(\beta>\alpha)$ and $\gamma=0.001$. Tables 6-9 display the results for Case 2 at $t=1.0$ and $t=10.0$.

In Case 3, parameters are chosen as $\alpha=0.5(\beta>>\alpha), \beta=10.0$ and $\gamma=0.001$. Tables 10-13 display the results for Case 3 at $t=1.0$ and $t=10.0$.

In Case 4, parameters are chosen as $\alpha=2.0(\alpha>\beta), \beta=0.5$ and $\gamma=0.001$. Tables 14-17 display the results for Case 4 at $t=1.0$ and $t=10.0$.

In Case 5, parameters are chosen as $\alpha=\beta=0.5$ and $\gamma=0.5$. Tables 18-21 display the results for Case 5 at $t=1.0$ and $t=10.0$.

In Case 6, parameters are chosen as $\alpha=0.5, \beta=2.0(\beta>\alpha)$ and $\gamma=0.5$. Tables 22-25 display the results for Case 6 at $t=1.0$ and $t=10.0$.

In Case 7, parameters are chosen as $\alpha=0.5(\beta>>\alpha), \beta=10.0$ and $\gamma=0.5$. Tables 26-29 display the results for Case 7 at $t=1.0$ and $t=10.0$.

In Case 8, parameters are chosen as $\alpha=2.0(\alpha>\beta), \beta=0.5$ and $\gamma=0.5$. Tables 30-33 display the results for Case 8 at $t=1.0$ and $t=10.0$.

In Case 9, we compare the absolute errors obtained by our four methods with other methods in $[13,14]$. Parameters are chosen as $\alpha=\beta=1.0$ and $\gamma=0.001$. Table 34 display the results for Case 9 at $t=0.05$, $t=0.1$ and $t=1.0$.

In Cases 10 and 11, we compare the accuracy of the fully implicit exponential scheme by chosen parameters as $\alpha=0.5, \beta=0.5(\beta=\alpha), \gamma=0.001$ and $k=0.1$ and $\alpha=0.5, \beta=10.0(\beta>>\alpha)$, $\gamma=0.5$ and $k=0.1$, respectively. Tables 35-38 display the results for Cases 10 and 11 at $t=1.0$ and $t=10.0$. We note that, for Cases $1-9, h=0.1$ and $k=0.005$.

Case 1: $\alpha=\beta=0.5$ and $\gamma=0.001$.

Table 2. A comparison between the exact and the numerical solutions at some values of $x$.

\begin{tabular}{ccccccc}
\hline $\boldsymbol{t}$ & $\boldsymbol{x}$ & Exact & NSFD1 & NSFD2 & EEFDM & FIEFDM \\
\hline 1 & 0.1 & $5.000858 \times 10^{-4}$ & $5.000764 \times 10^{-4}$ & $5.000764 \times 10^{-4}$ & $5.000768 \times 10^{-4}$ & $5.000769 \times 10^{-4}$ \\
& 0.5 & $5.001249 \times 10^{-4}$ & $5.000985 \times 10^{-4}$ & $5.000985 \times 10^{-4}$ & $5.000998 \times 10^{-4}$ & $5.000998 \times 10^{-4}$ \\
& 0.9 & $5.001640 \times 10^{-4}$ & $5.001545 \times 10^{-4}$ & $5.001545 \times 10^{-4}$ & $5.001549 \times 10^{-4}$ & $5.001549 \times 10^{-4}$ \\
10 & 0.1 & $5.007711 \times 10^{-4}$ & $5.007616 \times 10^{-4}$ & $5.007616 \times 10^{-4}$ & $5.007621 \times 10^{-4}$ & $5.007621 \times 10^{-4}$ \\
& 0.5 & $5.008102 \times 10^{-4}$ & $5.007838 \times 10^{-4}$ & $5.007838 \times 10^{-4}$ & $5.007851 \times 10^{-4}$ & $5.007851 \times 10^{-4}$ \\
& 0.9 & $5.008492 \times 10^{-4}$ & $5.008397 \times 10^{-4}$ & $5.008397 \times 10^{-4}$ & $5.008402 \times 10^{-4}$ & $5.008402 \times 10^{-4}$ \\
\hline
\end{tabular}


Table 3. The absolute errors at some values of $x$ for each of the numerical schemes.

\begin{tabular}{cccccc}
\hline$t$ & $x$ & NSFD1 & NSFD2 & EEFDM & FIEFDM \\
\hline 1 & 0.1 & $9.509935 \times 10^{-9}$ & $9.509930 \times 10^{-9}$ & $9.048885 \times 10^{-9}$ & $9.048222 \times 10^{-9}$ \\
& 0.5 & $2.641711 \times 10^{-8}$ & $2.641710 \times 10^{-8}$ & $2.513647 \times 10^{-8}$ & $2.513446 \times 10^{-8}$ \\
& 0.9 & $9.510585 \times 10^{-9}$ & $9.510580 \times 10^{-9}$ & $9.049488 \times 10^{-9}$ & $9.048757 \times 10^{-9}$ \\
10 & 0.1 & $9.510514 \times 10^{-9}$ & $9.510509 \times 10^{-9}$ & $9.049214 \times 10^{-9}$ & $9.048760 \times 10^{-9}$ \\
& 0.5 & $2.641900 \times 10^{-8}$ & $2.641898 \times 10^{-8}$ & $2.513754 \times 10^{-8}$ & $2.513624 \times 10^{-8}$ \\
& 0.9 & $9.511164 \times 10^{-9}$ & $9.511159 \times 10^{-9}$ & $9.049817 \times 10^{-9}$ & $9.049302 \times 10^{-9}$ \\
\hline
\end{tabular}

Table 4. The relative errors at some values of $x$ for each of the numerical schemes.

\begin{tabular}{cccccc}
\hline$t$ & $x$ & NSFD1 & NSFD2 & EEFDM & FIEFDM \\
\hline 1 & 0.1 & $1.901660 \times 10^{-5}$ & $1.901659 \times 10^{-5}$ & $1.809466 \times 10^{-5}$ & $1.809333 \times 10^{-5}$ \\
& 0.5 & $5.282102 \times 10^{-5}$ & $5.282100 \times 10^{-5}$ & $5.026038 \times 10^{-5}$ & $5.025637 \times 10^{-5}$ \\
& 0.9 & $1.901493 \times 10^{-5}$ & $1.901492 \times 10^{-5}$ & $1.809304 \times 10^{-5}$ & $1.809158 \times 10^{-5}$ \\
10 & 0.1 & $1.899174 \times 10^{-5}$ & $1.899173 \times 10^{-5}$ & $1.807056 \times 10^{-5}$ & $1.806967 \times 10^{-5}$ \\
& 0.5 & $5.275251 \times 10^{-5}$ & $5.275249 \times 10^{-5}$ & $5.019375 \times 10^{-5}$ & $5.019115 \times 10^{-5}$ \\
& 0.9 & $1.899007 \times 10^{-5}$ & $1.899006 \times 10^{-5}$ & $1.806894 \times 10^{-5}$ & $1.806792 \times 10^{-5}$ \\
\hline
\end{tabular}

Table 5. $L_{1}$ and $L_{\infty}$ error norms with CPU time taken for the four numerical methods.

\begin{tabular}{ccccc}
\hline $\boldsymbol{t}$ & Schemes & $\boldsymbol{L}_{\mathbf{1}}$ Error & $\boldsymbol{L}_{\boldsymbol{\infty}}$ Error & CPU Time \\
\hline 1 & NSFD1 & $1.743535 \times 10^{-8}$ & $2.641711 \times 10^{-8}$ & 0.0642 \\
& NSFD2 & $1.743534 \times 10^{-8}$ & $2.641710 \times 10^{-8}$ & 0.0649 \\
& EEFDM & $1.659010 \times 10^{-8}$ & $2.513647 \times 10^{-8}$ & 0.0660 \\
& FIEFDM & $1.658878 \times 10^{-8}$ & $2.513446 \times 10^{-8}$ & 0.0683 \\
10 & NSFD1 & $1.743654 \times 10^{-8}$ & $2.641900 \times 10^{-8}$ & 0.2261 \\
& NSFD2 & $1.743653 \times 10^{-8}$ & $2.641898 \times 10^{-8}$ & 0.2247 \\
& EEFDM & $1.659078 \times 10^{-8}$ & $2.513754 \times 10^{-8}$ & 0.2210 \\
& FIEFDM & $1.658990 \times 10^{-8}$ & $2.513624 \times 10^{-8}$ & 0.2341 \\
\hline
\end{tabular}

Case 2: $\alpha=0.5, \beta=2.0(\beta>\alpha)$ and $\gamma=0.001$

Table 6. A comparison between the exact and the numerical solutions at some values of $x$.

\begin{tabular}{ccccccc}
\hline $\boldsymbol{t}$ & $\boldsymbol{x}$ & Exact & NSFD1 & NSFD2 & EEFDM & FIEFDM \\
\hline 1 & 0.1 & $5.004115 \times 10^{-4}$ & $5.003626 \times 10^{-4}$ & $5.003626 \times 10^{-4}$ & $5.003715 \times 10^{-4}$ & $5.003715 \times 10^{-4}$ \\
& 0.5 & $5.004997 \times 10^{-4}$ & $5.003639 \times 10^{-4}$ & $5.003639 \times 10^{-4}$ & $5.003886 \times 10^{-4}$ & $5.003886 \times 10^{-4}$ \\
& 0.9 & $5.005880 \times 10^{-4}$ & $5.005391 \times 10^{-4}$ & $5.005391 \times 10^{-4}$ & $5.005480 \times 10^{-4}$ & $5.005480 \times 10^{-4}$ \\
10 & 0.1 & $5.039160 \times 10^{-4}$ & $5.038671 \times 10^{-4}$ & $5.038671 \times 10^{-4}$ & $5.038760 \times 10^{-4}$ & $5.038760 \times 10^{-4}$ \\
& 0.5 & $5.040428 \times 10^{-4}$ & $5.038684 \times 10^{-4}$ & $5.038684 \times 10^{-4}$ & $5.038931 \times 10^{-4}$ & $5.038931 \times 10^{-4}$ \\
& 0.9 & $5.040926 \times 10^{-4}$ & $5.040436 \times 10^{-4}$ & $5.040436 \times 10^{-4}$ & $5.040525 \times 10^{-4}$ & $5.040525 \times 10^{-4}$ \\
\hline
\end{tabular}

Table 7. The absolute errors at some values of $x$ for each of the numerical schemes.

\begin{tabular}{cccccc}
\hline$t$ & $x$ & NSFD1 & NSFD2 & EEFDM & FIEFDM \\
\hline 1 & 0.1 & $4.891509 \times 10^{-8}$ & $4.891498 \times 10^{-8}$ & $4.001139 \times 10^{-8}$ & $4.000776 \times 10^{-8}$ \\
& 0.5 & $1.358748 \times 10^{-7}$ & $1.358745 \times 10^{-7}$ & $1.111457 \times 10^{-7}$ & $1.111355 \times 10^{-7}$ \\
& 0.9 & $4.891869 \times 10^{-8}$ & $4.891858 \times 10^{-8}$ & $4.001405 \times 10^{-8}$ & $4.000958 \times 10^{-8}$ \\
10 & 0.1 & $4.892413 \times 10^{-8}$ & $4.892402 \times 10^{-8}$ & $4.001053 \times 10^{-8}$ & $4.000788 \times 10^{-8}$ \\
& 0.5 & $1.359053 \times 10^{-7}$ & $1.359050 \times 10^{-7}$ & $1.111440 \times 10^{-7}$ & $1.111369 \times 10^{-7}$ \\
& 0.9 & $4.892767 \times 10^{-8}$ & $4.892756 \times 10^{-8}$ & $4.001314 \times 10^{-8}$ & $4.000966 \times 10^{-8}$ \\
\hline
\end{tabular}


Table 8. The relative errors at some values of $x$ for each of the numerical schemes.

\begin{tabular}{cccccc}
\hline$t$ & $x$ & NSFD1 & NSFD2 & EEFDM & FIEFDM \\
\hline 1 & 0.1 & $9.774973 \times 10^{-5}$ & $9.774951 \times 10^{-5}$ & $7.995698 \times 10^{-5}$ & $7.994972 \times 10^{-5}$ \\
& 0.5 & $2.714782 \times 10^{-4}$ & $2.714776 \times 10^{-4}$ & $2.220695 \times 10^{-4}$ & $2.220490 \times 10^{-4}$ \\
& 0.9 & $9.772245 \times 10^{-5}$ & $9.772223 \times 10^{-5}$ & $7.993409 \times 10^{-5}$ & $7.992517 \times 10^{-5}$ \\
10 & 0.1 & $9.708786 \times 10^{-5}$ & $9.708764 \times 10^{-5}$ & $7.939920 \times 10^{-5}$ & $7.939394 \times 10^{-5}$ \\
& 0.5 & $2.696512 \times 10^{-4}$ & $2.696506 \times 10^{-4}$ & $2.205220 \times 10^{-4}$ & $2.205079 \times 10^{-4}$ \\
& 0.9 & $9.706089 \times 10^{-5}$ & $9.706068 \times 10^{-5}$ & $7.937658 \times 10^{-5}$ & $7.936967 \times 10^{-5}$ \\
\hline
\end{tabular}

Table 9. $L_{1}$ and $L_{\infty}$ error norms with CPU time taken for the four numerical methods.

\begin{tabular}{ccccc}
\hline $\boldsymbol{t}$ & Schemes & $\boldsymbol{L}_{\mathbf{1}}$ Error & $\boldsymbol{L}_{\infty}$ Error & CPU Time (Sec) \\
\hline 1 & NSFD1 & $8.967845 \times 10^{-8}$ & $1.358748 \times 10^{-7}$ & 0.0641 \\
& NSFD2 & $8.967825 \times 10^{-8}$ & $1.358745 \times 10^{-7}$ & 0.0643 \\
& EEFDM & $7.335633 \times 10^{-8}$ & $1.111457 \times 10^{-7}$ & 0.0645 \\
& FIEFDM & $7.334929 \times 10^{-8}$ & $1.111355 \times 10^{-7}$ & 0.0654 \\
10 & NSFD1 & $8.969751 \times 10^{-8}$ & $1.359053 \times 10^{-7}$ & 0.2322 \\
& NSFD2 & $8.969731 \times 10^{-8}$ & $1.359053 \times 10^{-7}$ & 0.2273 \\
& EEFDM & $7.335505 \times 10^{-8}$ & $1.111440 \times 10^{-7}$ & 0.2237 \\
& FIEFDM & $7.335000 \times 10^{-8}$ & $1.111369 \times 10^{-7}$ & 0.2321 \\
\hline
\end{tabular}

Case 3: $\alpha=0.5, \beta=10.0(\beta>>\alpha)$, and $\gamma=0.001$. (singularly perturbed)

Table 10. A comparison between the exact and the numerical solutions at some values of $x$.

\begin{tabular}{ccccccc}
\hline$t$ & $x$ & Exact & NSFD1 & NSFD2 & EEFDM & FIEFDM \\
\hline 1 & 0.1 & $5.022873 \times 10^{-4}$ & $5.016837 \times 10^{-4}$ & $5.016837 \times 10^{-4}$ & $5.020743 \times 10^{-4}$ & $5.020743 \times 10^{-4}$ \\
& 0.5 & $5.024987 \times 10^{-4}$ & $5.008301 \times 10^{-4}$ & $5.008301 \times 10^{-4}$ & $5.019071 \times 10^{-4}$ & $5.019071 \times 10^{-4}$ \\
& 0.9 & $5.027102 \times 10^{-4}$ & $5.021065 \times 10^{-4}$ & $5.021065 \times 10^{-4}$ & $5.024972 \times 10^{-4}$ & $5.024972 \times 10^{-4}$ \\
10 & 0.1 & $5.223822 \times 10^{-4}$ & $5.217618 \times 10^{-4}$ & $5.217619 \times 10^{-4}$ & $5.221696 \times 10^{-4}$ & $5.221696 \times 10^{-4}$ \\
& 0.5 & $5.225932 \times 10^{-4}$ & $5.208699 \times 10^{-4}$ & $5.208699 \times 10^{-4}$ & $5.220027 \times 10^{-4}$ & $5.220026 \times 10^{-4}$ \\
& 0.9 & $5.228042 \times 10^{-4}$ & $5.221838 \times 10^{-4}$ & $5.221838 \times 10^{-4}$ & $5.225917 \times 10^{-4}$ & $5.225916 \times 10^{-4}$ \\
\hline
\end{tabular}

Table 11. The absolute errors at some values of $x$ for each of the numerical schemes.

\begin{tabular}{cccccc}
\hline$t$ & $x$ & NSFD1 & NSFD2 & EEFDM & FIEFDM \\
\hline 1 & 0.1 & $6.036257 \times 10^{-7}$ & $6.036201 \times 10^{-7}$ & $2.129791 \times 10^{-7}$ & $2.129933 \times 10^{-7}$ \\
& 0.5 & $1.668652 \times 10^{-6}$ & $1.668637 \times 10^{-6}$ & $5.916245 \times 10^{-7}$ & $5.917030 \times 10^{-7}$ \\
& 0.9 & $6.036927 \times 10^{-7}$ & $6.036870 \times 10^{-7}$ & $2.129928 \times 10^{-7}$ & $2.129952 \times 10^{-7}$ \\
10 & 0.1 & $6.203521 \times 10^{-7}$ & $6.203444 \times 10^{-7}$ & $2.125747 \times 10^{-7}$ & $2.125934 \times 10^{-7}$ \\
& 0.5 & $1.723336 \times 10^{-6}$ & $1.723315 \times 10^{-6}$ & $5.905050 \times 10^{-7}$ & $5.905967 \times 10^{-7}$ \\
& 0.9 & $6.204109 \times 10^{-7}$ & $6.204031 \times 10^{-7}$ & $2.125841 \times 10^{-7}$ & $2.125916 \times 10^{-7}$ \\
\hline
\end{tabular}

Table 12. The relative errors at some values of $x$ for each of the numerical schemes.

\begin{tabular}{cccccc}
\hline$t$ & $x$ & NSFD1 & NSFD2 & EEFDM & FIEFDM \\
\hline 1 & 0.1 & $1.201754 \times 10^{-3}$ & $1.201743 \times 10^{-3}$ & $4.240185 \times 10^{-4}$ & $4.240467 \times 10^{-4}$ \\
& 0.5 & $3.320709 \times 10^{-3}$ & $3.320679 \times 10^{-3}$ & $1.177365 \times 10^{-3}$ & $1.177521 \times 10^{-3}$ \\
& 0.9 & $1.200876 \times 10^{-3}$ & $1.200865 \times 10^{-3}$ & $4.236891 \times 10^{-4}$ & $4.236939 \times 10^{-4}$ \\
10 & 0.1 & $1.187545 \times 10^{-3}$ & $1.187530 \times 10^{-3}$ & $4.069333 \times 10^{-4}$ & $4.069691 \times 10^{-4}$ \\
& 0.5 & $3.297662 \times 10^{-3}$ & $3.297621 \times 10^{-3}$ & $1.129952 \times 10^{-3}$ & $1.130127 \times 10^{-3}$ \\
& 0.9 & $1.186698 \times 10^{-3}$ & $1.186683 \times 10^{-3}$ & $4.066228 \times 10^{-4}$ & $4.066371 \times 10^{-4}$ \\
\hline
\end{tabular}


Table 13. $L_{1}$ and $L_{\infty}$ error norms with CPU time taken for the four numerical methods.

\begin{tabular}{ccccc}
\hline $\boldsymbol{t}$ & Schemes & $\boldsymbol{L}_{\mathbf{1}}$ Error & $\boldsymbol{L}_{\boldsymbol{\infty}}$ Error & CPU Time $(\mathrm{Sec})$ \\
\hline 1 & NSFD1 & $1.102963 \times 10^{-6}$ & $1.668652 \times 10^{-6}$ & 0.0475 \\
& NSFD2 & $1.102954 \times 10^{-6}$ & $1.668637 \times 10^{-6}$ & 0.0748 \\
& EEFDM & $3.904728 \times 10^{-7}$ & $4.320404 \times 10^{-7}$ & 0.1122 \\
& FIEFDM & $3.905126 \times 10^{-7}$ & $4.320876 \times 10^{-7}$ & 0.1620 \\
10 & NSFD1 & $1.137391 \times 10^{-6}$ & $1.723336 \times 10^{-6}$ & 0.2023 \\
& NSFD2 & $1.137376 \times 10^{-6}$ & $1.723315 \times 10^{-6}$ & 0.3251 \\
& EEFDM & $3.897320 \times 10^{-7}$ & $4.312213 \times 10^{-7}$ & 0.2729 \\
& FIEFDM & $3.897805 \times 10^{-7}$ & $5.905967 \times 10^{-7}$ & 0.3018 \\
\hline
\end{tabular}

Case 4: $\alpha=2.0(\alpha>\beta), \beta=0.5$ and $\gamma=0.001$.

Table 14. A comparison between the exact and the numerical solutions at some values of $x$.

\begin{tabular}{|c|c|c|c|c|c|c|}
\hline$t$ & $x$ & Exact & NSFD1 & NSFD2 & EEFDM & FIEFDM \\
\hline \multirow[t]{3}{*}{1} & 0.1 & $5.000267 \times 10^{-4}$ & $5.000196 \times 10^{-4}$ & $5.000196 \times 10^{-4}$ & $5.000200 \times 10^{-4}$ & $5.000200 \times 10^{-4}$ \\
\hline & 0.5 & $5.000473 \times 10^{-4}$ & $5.000280 \times 10^{-4}$ & $5.000280 \times 10^{-4}$ & $5.000290 \times 10^{-4}$ & $5.000290 \times 10^{-4}$ \\
\hline & 0.9 & $5.000680 \times 10^{-4}$ & $5.000611 \times 10^{-4}$ & $5.000611 \times 10^{-4}$ & $5.000614 \times 10^{-4}$ & $5.000614 \times 10^{-4}$ \\
\hline \multirow[t]{3}{*}{10} & 0.1 & $5.002138 \times 10^{-4}$ & $5.002121 \times 10^{-4}$ & $5.002121 \times 10^{-4}$ & $5.002124 \times 10^{-4}$ & $5.002124 \times 10^{-4}$ \\
\hline & 0.5 & $5.002397 \times 10^{-4}$ & $5.002205 \times 10^{-4}$ & $5.002205 \times 10^{-4}$ & $5.002214 \times 10^{-4}$ & $5.002214 \times 10^{-4}$ \\
\hline & 0.9 & $5.002604 \times 10^{-4}$ & $5.002535 \times 10^{-4}$ & $5.002535 \times 10^{-4}$ & $5.002539 \times 10^{-4}$ & $5.002538 \times 10^{-4}$ \\
\hline
\end{tabular}

Table 15. The absolute errors at some values of $x$ for each of the numerical schemes.

\begin{tabular}{cccccc}
\hline$t$ & $x$ & NSFD1 & NSFD2 & EEFDM & FIEFDM \\
\hline 1 & 0.1 & $6.922896 \times 10^{-9}$ & $6.922895 \times 10^{-9}$ & $6.585668 \times 10^{-9}$ & $6.585338 \times 10^{-9}$ \\
& 0.5 & $1.923270 \times 10^{-8}$ & $1.923269 \times 10^{-8}$ & $1.829585 \times 10^{-8}$ & $1.829491 \times 10^{-8}$ \\
& 0.9 & $6.924789 \times 10^{-9}$ & $6.924788 \times 10^{-9}$ & $6.587425 \times 10^{-9}$ & $6.587092 \times 10^{-9}$ \\
10 & 0.1 & $6.923333 \times 10^{-9}$ & $6.923332 \times 10^{-9}$ & $6.585922 \times 10^{-9}$ & $6.585749 \times 10^{-9}$ \\
& 0.5 & $1.923411 \times 10^{-8}$ & $1.923411 \times 10^{-8}$ & $1.829667 \times 10^{-8}$ & $1.829624 \times 10^{-8}$ \\
& 0.9 & $6.925227 \times 10^{-9}$ & $6.925226 \times 10^{-9}$ & $6.587680 \times 10^{-9}$ & $6.587504 \times 10^{-9}$ \\
\hline
\end{tabular}

Table 16. The relative errors at some values of $x$ for each of the numerical schemes.

\begin{tabular}{cccccc}
\hline$t$ & $x$ & NSFD1 & NSFD2 & EEFDM & FIEFDM \\
\hline 1 & 0.1 & $1.384506 \times 10^{-5}$ & $1.384505 \times 10^{-5}$ & $1.317064 \times 10^{-5}$ & $1.316998 \times 10^{-5}$ \\
& 0.5 & $3.846175 \times 10^{-5}$ & $3.846175 \times 10^{-5}$ & $3.658823 \times 10^{-5}$ & $3.658636 \times 10^{-5}$ \\
& 0.9 & $1.384770 \times 10^{-5}$ & $1.384769 \times 10^{-5}$ & $1.317306 \times 10^{-5}$ & $1.317239 \times 10^{-5}$ \\
10 & 0.1 & $1.384060 \times 10^{-5}$ & $1.384060 \times 10^{-5}$ & $1.316608 \times 10^{-5}$ & $1.316573 \times 10^{-5}$ \\
& 0.5 & $3.844979 \times 10^{-5}$ & $3.844978 \times 10^{-5}$ & $3.657580 \times 10^{-5}$ & $3.657494 \times 10^{-5}$ \\
& 0.9 & $1.384324 \times 10^{-5}$ & $1.384324 \times 10^{-5}$ & $1.316850 \times 10^{-5}$ & $1.316815 \times 10^{-5}$ \\
\hline
\end{tabular}

Table 17. $L_{1}$ and $L_{\infty}$ error norms with CPU time taken for the four numerical methods.

\begin{tabular}{ccccc}
\hline $\boldsymbol{t}$ & Schemes & $\boldsymbol{L}_{\mathbf{1}}$ Error & $\boldsymbol{L}_{\infty}$ Error & CPU Time $(\mathrm{Sec})$ \\
\hline 1 & NSFD1 & $1.269362 \times 10^{-8}$ & $1.923270 \times 10^{-8}$ & 0.0654 \\
& NSFD2 & $1.269362 \times 10^{-8}$ & $1.923269 \times 10^{-8}$ & 0.0677 \\
& EEFDM & $1.207528 \times 10^{-8}$ & $1.829585 \times 10^{-8}$ & 0.0677 \\
& FIEFDM & $1.207467 \times 10^{-8}$ & $1.829491 \times 10^{-8}$ & 0.0688 \\
10 & NSFD1 & $1.269451 \times 10^{-8}$ & $1.923411 \times 10^{-8}$ & 0.2381 \\
& NSFD2 & $1.269451 \times 10^{-8}$ & $1.923411 \times 10^{-8}$ & 0.2354 \\
& EEFDM & $1.207580 \times 10^{-8}$ & $1.829667 \times 10^{-8}$ & 0.2242 \\
& FIEFDM & $1.207551 \times 10^{-8}$ & $1.829624 \times 10^{-8}$ & 0.2372 \\
\hline
\end{tabular}


Case 5: $\alpha=\beta=0.5$ and $\gamma=0.5$

Table 18. A comparison between the exact and the numerical solutions at some values of $x$.

\begin{tabular}{ccccccc}
\hline$t$ & $x$ & Exact & NSFD1 & NSFD2 & EEFDM & FIEFDM \\
\hline 1 & 0.1 & $2.636642 \times 10^{-1}$ & $2.619319 \times 10^{-1}$ & $2.619323 \times 10^{-1}$ & $2.620016 \times 10^{-1}$ & $2.620423 \times 10^{-1}$ \\
& 0.5 & $2.733691 \times 10^{-1}$ & $2.684776 \times 10^{-1}$ & $2.684788 \times 10^{-1}$ & $2.686755 \times 10^{-1}$ & $2.688005 \times 10^{-1}$ \\
& 0.9 & $2.830035 \times 10^{-1}$ & $2.812149 \times 10^{-1}$ & $2.812153 \times 10^{-1}$ & $2.812875 \times 10^{-1}$ & $2.813362 \times 10^{-1}$ \\
10 & 0.1 & $3.573732 \times 10^{-1}$ & $3.559870 \times 10^{-1}$ & $3.559874 \times 10^{-1}$ & $3.560367 \times 10^{-1}$ & $3.560550 \times 10^{-1}$ \\
& 0.5 & $3.651974 \times 10^{-1}$ & $3.612915 \times 10^{-1}$ & $3.612927 \times 10^{-1}$ & $3.614329 \times 10^{-1}$ & $3.614903 \times 10^{-1}$ \\
& 0.9 & $3.727452 \times 10^{-1}$ & $3.713231 \times 10^{-1}$ & $3.713236 \times 10^{-1}$ & $3.713749 \times 10^{-1}$ & $3.713974 \times 10^{-1}$ \\
\hline
\end{tabular}

Table 19. The absolute errors at some values of $x$ for each of the numerical schemes.

\begin{tabular}{cccccc}
\hline$t$ & $x$ & NSFD1 & NSFD2 & EEFDM & FIEFDM \\
\hline 1 & 0.1 & $1.732294 \times 10^{-3}$ & $1.731870 \times 10^{-3}$ & $1.662559 \times 10^{-3}$ & $1.621813 \times 10^{-3}$ \\
& 0.5 & $4.891452 \times 10^{-3}$ & $4.890247 \times 10^{-3}$ & $4.693599 \times 10^{-3}$ & $4.568532 \times 10^{-3}$ \\
& 0.9 & $1.788565 \times 10^{-3}$ & $1.788119 \times 10^{-3}$ & $1.715994 \times 10^{-3}$ & $1.667276 \times 10^{-3}$ \\
10 & 0.1 & $1.386199 \times 10^{-3}$ & $1.385769 \times 10^{-3}$ & $1.336481 \times 10^{-3}$ & $1.318209 \times 10^{-3}$ \\
& 0.5 & $3.905879 \times 10^{-3}$ & $3.904660 \times 10^{-3}$ & $3.764468 \times 10^{-3}$ & $3.707117 \times 10^{-3}$ \\
& 0.9 & $1.422126 \times 10^{-3}$ & $1.421680 \times 10^{-3}$ & $1.370378 \times 10^{-3}$ & $1.347886 \times 10^{-3}$ \\
\hline
\end{tabular}

Table 20. The relative errors at some values of $x$ for each of the numerical schemes.

\begin{tabular}{cccccc}
\hline$t$ & $x$ & NSFD1 & NSFD2 & EEFDM & FIEFDM \\
\hline 1 & 0.1 & $6.570078 \times 10^{-3}$ & $6.568471 \times 10^{-3}$ & $6.305595 \times 10^{-3}$ & $6.151057 \times 10^{-3}$ \\
& 0.5 & $1.789322 \times 10^{-2}$ & $1.788881 \times 10^{-2}$ & $1.716946 \times 10^{-2}$ & $1.671196 \times 10^{-2}$ \\
& 0.9 & $6.319940 \times 10^{-3}$ & $6.318365 \times 10^{-3}$ & $6.063510 \times 10^{-3}$ & $5.891364 \times 10^{-3}$ \\
10 & 0.1 & $3.878856 \times 10^{-3}$ & $3.877653 \times 10^{-3}$ & $3.739734 \times 10^{-3}$ & $3.688607 \times 10^{-3}$ \\
& 0.5 & $1.069525 \times 10^{-2}$ & $1.069191 \times 10^{-2}$ & $1.030804 \times 10^{-2}$ & $1.015099 \times 10^{-2}$ \\
& 0.9 & $3.815276 \times 10^{-3}$ & $3.814081 \times 10^{-3}$ & $3.676447 \times 10^{-3}$ & $3.616104 \times 10^{-3}$ \\
\hline
\end{tabular}

Table 21. $L_{1}$ and $L_{\infty}$ error norms with CPU times for the four numerical methods.

\begin{tabular}{ccccc}
\hline $\boldsymbol{t}$ & Schemes & $\boldsymbol{L}_{\mathbf{1}}$ Error & $\boldsymbol{L}_{\boldsymbol{\infty}}$ Error & CPU Time (Sec) \\
\hline 1 & NSFD1 & $3.228089 \times 10^{-3}$ & $4.891452 \times 10^{-3}$ & 0.0621 \\
& NSFD2 & $3.227293 \times 10^{-3}$ & $4.890247 \times 10^{-3}$ & 0.0637 \\
& EEFDM & $3.097547 \times 10^{-3}$ & $4.693599 \times 10^{-3}$ & 0.0659 \\
& FIEFDM & $3.014506 \times 10^{-3}$ & $4.568532 \times 10^{-3}$ & 0.0671 \\
10 & NSFD1 & $2.576806 \times 10^{-3}$ & $3.905879 \times 10^{-3}$ & 0.2335 \\
& NSFD2 & $2.576002 \times 10^{-3}$ & $3.904660 \times 10^{-3}$ & 0.2281 \\
& EEFDM & $2.483571 \times 10^{-3}$ & $3.764468 \times 10^{-3}$ & 0.2328 \\
& FIEFDM & $2.445540 \times 10^{-3}$ & $3.707117 \times 10^{-3}$ & 0.2871 \\
\hline
\end{tabular}

Case 6: $\alpha=0.5, \beta=2.0(\beta>\alpha)$ and $\gamma=0.5$.

Table 22. A comparison between the exact and the numerical solutions at some values of $x$.

\begin{tabular}{ccccccc}
\hline $\boldsymbol{t}$ & $\boldsymbol{x}$ & Exact & NSFD1 & NSFD2 & EEFDM & FIEFDM \\
\hline 1 & 0.1 & $3.197787 \times 10^{-1}$ & $3.115395 \times 10^{-1}$ & $3.115521 \times 10^{-1}$ & $3.129688 \times 10^{-1}$ & $3.131647 \times 10^{-1}$ \\
& 0.5 & $3.395893 \times 10^{-1}$ & $3.164245 \times 10^{-1}$ & $3.164602 \times 10^{-1}$ & $3.204963 \times 10^{-1}$ & $3.210964 \times 10^{-1}$ \\
& 0.9 & $3.581881 \times 10^{-1}$ & $3.498705 \times 10^{-1}$ & $3.498835 \times 10^{-1}$ & $3.513357 \times 10^{-1}$ & $3.515904 \times 10^{-1}$ \\
10 & 0.1 & $4.976668 \times 10^{-1}$ & $4.974441 \times 10^{-1}$ & $4.974445 \times 10^{-1}$ & $4.974708 \times 10^{-1}$ & $4.974707 \times 10^{-1}$ \\
& 0.5 & $4.979926 \times 10^{-1}$ & $4.975407 \times 10^{-1}$ & $4.975418 \times 10^{-1}$ & $4.976175 \times 10^{-1}$ & $4.976172 \times 10^{-1}$ \\
& 0.9 & $4.983164 \times 10^{-1}$ & $4.981586 \times 10^{-1}$ & $4.981589 \times 10^{-1}$ & $4.981856 \times 10^{-1}$ & $4.981855 \times 10^{-1}$ \\
\hline
\end{tabular}


Table 23. The absolute errors at some values of $x$ for each of the numerical schemes.

\begin{tabular}{cccccc}
\hline$t$ & $x$ & NSFD1 & NSFD2 & EEFDM & FIEFDM \\
\hline 1 & 0.1 & $8.239162 \times 10^{-3}$ & $8.226605 \times 10^{-3}$ & $6.809932 \times 10^{-3}$ & $6.613999 \times 10^{-3}$ \\
& 0.5 & $2.316489 \times 10^{-2}$ & $2.312918 \times 10^{-2}$ & $1.909310 \times 10^{-2}$ & $1.849292 \times 10^{-2}$ \\
& 0.9 & $8.317574 \times 10^{-3}$ & $8.304529 \times 10^{-3}$ & $6.852408 \times 10^{-3}$ & $6.597658 \times 10^{-3}$ \\
10 & 0.1 & $1.626796 \times 10^{-4}$ & $1.622971 \times 10^{-4}$ & $1.360407 \times 10^{-4}$ & $1.361553 \times 10^{-4}$ \\
& 0.5 & $4.518804 \times 10^{-4}$ & $4.507901 \times 10^{-4}$ & $3.751243 \times 10^{-4}$ & $3.754311 \times 10^{-4}$ \\
& 0.9 & $1.578207 \times 10^{-4}$ & $1.574438 \times 10^{-4}$ & $1.308204 \times 10^{-4}$ & $1.309152 \times 10^{-4}$ \\
\hline
\end{tabular}

Table 24. The relative errors at some values of $x$ for each of the numerical schemes.

\begin{tabular}{cccccc}
\hline$t$ & $x$ & NSFD1 & NSFD2 & EEFDM & FIEFDM \\
\hline 1 & 0.1 & $2.576520 \times 10^{-2}$ & $2.572593 \times 10^{-2}$ & $2.129576 \times 10^{-2}$ & $2.068305 \times 10^{-2}$ \\
& 0.5 & $6.821441 \times 10^{-2}$ & $6.810926 \times 10^{-2}$ & $5.622408 \times 10^{-2}$ & $5.445674 \times 10^{-2}$ \\
& 0.9 & $2.322125 \times 10^{-2}$ & $2.318483 \times 10^{-2}$ & $1.913075 \times 10^{-2}$ & $1.841954 \times 10^{-2}$ \\
10 & 0.1 & $3.269239 \times 10^{-4}$ & $3.261553 \times 10^{-4}$ & $2.733899 \times 10^{-4}$ & $2.736201 \times 10^{-4}$ \\
& 0.5 & $9.074039 \times 10^{-4}$ & $9.052145 \times 10^{-4}$ & $7.532729 \times 10^{-4}$ & $7.538889 \times 10^{-4}$ \\
& 0.9 & $3.167078 \times 10^{-4}$ & $3.159516 \times 10^{-4}$ & $2.625249 \times 10^{-4}$ & $2.627150 \times 10^{-4}$ \\
\hline
\end{tabular}

Table 25. $L_{1}$ and $L_{\infty}$ error norms with CPU times for the four numerical methods.

\begin{tabular}{ccccc}
\hline $\boldsymbol{t}$ & Schemes & $\boldsymbol{L}_{\mathbf{1}}$ Error & $\boldsymbol{L}_{\boldsymbol{\infty}}$ Error & CPU Time $(\mathrm{Sec})$ \\
\hline 1 & NSFD1 & $1.525544 \times 10^{-2}$ & $2.316489 \times 10^{-2}$ & 0.0613 \\
& NSFD2 & $1.523190 \times 10^{-2}$ & $2.312918 \times 10^{-2}$ & 0.0625 \\
& EEFDM & $1.257824 \times 10^{-2}$ & $1.909310 \times 10^{-2}$ & 0.0626 \\
& FIEFDM & $1.217271 \times 10^{-2}$ & $1.849292 \times 10^{-2}$ & 0.0628 \\
10 & NSFD1 & $2.969031 \times 10^{-4}$ & $4.518804 \times 10^{-4}$ & 0.2268 \\
& NSFD2 & $2.961906 \times 10^{-4}$ & $4.507901 \times 10^{-4}$ & 0.2217 \\
& EEFDM & $2.466924 \times 10^{-4}$ & $3.751243 \times 10^{-4}$ & 0.2238 \\
& FIEFDM & $2.468907 \times 10^{-4}$ & $3.754311 \times 10^{-4}$ & 0.2401 \\
\hline
\end{tabular}

Case 7: $\alpha=0.5, \beta=10.0(\beta>>\alpha)$, and $\gamma=0.5$. (singularly perturbed)

Table 26. A comparison between the exact and the numerical solutions at some values of $x$.

\begin{tabular}{|c|c|c|c|c|c|c|}
\hline$t$ & $x$ & Exact & NSFD1 & NSFD2 & EEFDM & FIEFDM \\
\hline \multirow[t]{3}{*}{1} & 0.1 & $4.826732 \times 10^{-1}$ & $4.257945 \times 10^{-1}$ & $4.261428 \times 10^{-1}$ & $4.749299 \times 10^{-1}$ & $4.745925 \times 10^{-1}$ \\
\hline & 0.5 & $4.885113 \times 10^{-1}$ & $3.075586 \times 10^{-1}$ & $3.084981 \times 10^{-1}$ & $4.662423 \times 10^{-1}$ & $4.650936 \times 10^{-1}$ \\
\hline & 0.9 & $4.924132 \times 10^{-1}$ & $4.322566 \times 10^{-1}$ & $4.325748 \times 10^{-1}$ & $4.852025 \times 10^{-1}$ & $4.848678 \times 10^{-1}$ \\
\hline \multirow[t]{3}{*}{10} & 0.1 & $5.000000 \times 10^{-1}$ & $4.999761 \times 10^{-1}$ & $4.999801 \times 10^{-1}$ & $5.000000 \times 10^{-1}$ & $5.000000 \times 10^{-1}$ \\
\hline & 0.5 & $5.000000 \times 10^{-1}$ & $4.999159 \times 10^{-1}$ & $4.999301 \times 10^{-1}$ & $5.000000 \times 10^{-1}$ & $5.000000 \times 10^{-1}$ \\
\hline & 0.9 & $5.000000 \times 10^{-1}$ & $4.999717 \times 10^{-1}$ & $4.999765 \times 10^{-1}$ & $5.000000 \times 10^{-1}$ & $5.000000 \times 10^{-1}$ \\
\hline
\end{tabular}

Table 27. The absolute errors at some values of $x$ for each of the numerical schemes.

\begin{tabular}{cccccc}
\hline $\boldsymbol{t}$ & $\boldsymbol{x}$ & NSFD1 & NSFD2 & EEFDM & FIEFDM \\
\hline 1 & 0.1 & $5.687871 \times 10^{-2}$ & $5.653040 \times 10^{-2}$ & $7.743372 \times 10^{-3}$ & $8.080728 \times 10^{-3}$ \\
& 0.5 & $1.809527 \times 10^{-1}$ & $1.800132 \times 10^{-1}$ & $2.226902 \times 10^{-2}$ & $2.341770 \times 10^{-2}$ \\
& 0.9 & $6.015664 \times 10^{-2}$ & $5.983845 \times 10^{-2}$ & $7.210756 \times 10^{-3}$ & $7.545411 \times 10^{-3}$ \\
10 & 0.1 & $2.388699 \times 10^{-5}$ & $1.985583 \times 10^{-5}$ & $2.275957 \times 10^{-15}$ & $2.331470 \times 10^{-15}$ \\
& 0.5 & $8.408308 \times 10^{-5}$ & $6.989335 \times 10^{-5}$ & $6.716849 \times 10^{-15}$ & $6.772361 \times 10^{-15}$ \\
& 0.9 & $2.826313 \times 10^{-5}$ & $2.349352 \times 10^{-5}$ & $2.109424 \times 10^{-15}$ & $2.164932 \times 10^{-15}$ \\
\hline
\end{tabular}


Table 28. The relative errors at some values of $x$ for each of the numerical schemes.

\begin{tabular}{cccccc}
\hline$t$ & $x$ & NSFD1 & NSFD2 & EEFDM & FIEFDM \\
\hline 1 & 0.1 & $1.178410 \times 10^{-1}$ & $1.171194 \times 10^{-1}$ & $1.604268 \times 10^{-2}$ & $1.674161 \times 10^{-2}$ \\
& 0.5 & $3.704166 \times 10^{-1}$ & $3.684934 \times 10^{-1}$ & $4.558548 \times 10^{-2}$ & $4.793687 \times 10^{-2}$ \\
& 0.9 & $1.221670 \times 10^{-1}$ & $1.215208 \times 10^{-1}$ & $1.464371 \times 10^{-2}$ & $1.532333 \times 10^{-2}$ \\
10 & 0.1 & $4.777397 \times 10^{-5}$ & $3.971166 \times 10^{-5}$ & $4.551914 \times 10^{-15}$ & $4.662940 \times 10^{-15}$ \\
& 0.5 & $1.681662 \times 10^{-4}$ & $1.397867 \times 10^{-4}$ & $1.343370 \times 10^{-15}$ & $1.354472 \times 10^{-14}$ \\
& 0.9 & $5.652626 \times 10^{-5}$ & $4.698704 \times 10^{-5}$ & $4.218847 \times 10^{-15}$ & $4.329871 \times 10^{-15}$ \\
\hline
\end{tabular}

Table 29. $L_{1}$ and $L_{\infty}$ error norms with CPU time taken for the four numerical methods.

\begin{tabular}{ccccc}
\hline $\boldsymbol{t}$ & Schemes & $\boldsymbol{L}_{\mathbf{1}}$ Error & $\boldsymbol{L}_{\boldsymbol{\infty}}$ Error & CPU Time (Sec) \\
\hline 1 & NSFD1 & $1.158609 \times 10^{-1}$ & $1.809527 \times 10^{-1}$ & 0.0359 \\
& NSFD2 & $1.152403 \times 10^{-1}$ & $1.800132 \times 10^{-1}$ & 0.0325 \\
& EEFDM & $1.439775 \times 10^{-2}$ & $2.226902 \times 10^{-2}$ & 0.0311 \\
& FIEFDM & $1.511013 \times 10^{-2}$ & $2.341770 \times 10^{-2}$ & 0.0527 \\
10 & NSFD1 & $5.314160 \times 10^{-5}$ & $8.408308 \times 10^{-5}$ & 0.2114 \\
& NSFD2 & $4.417351 \times 10^{-5}$ & $6.989335 \times 10^{-5}$ & 0.1933 \\
& EEFDM & $4.352074 \times 10^{-15}$ & $6.716849 \times 10^{-15}$ & 0.2729 \\
& FIEFDM & $4.379830 \times 10^{-15}$ & $6.772360 \times 10^{-15}$ & 0.3112 \\
\hline
\end{tabular}

Case 8: $\alpha=2.0(\alpha>\beta), \beta=0.5$ and $\gamma=0.5$

Table 30. A comparison between the exact and the numerical solutions at some values of $x$.

\begin{tabular}{ccccccc}
\hline$t$ & $x$ & Exact & NSFD1 & NSFD2 & EEFDM & FIEFDM \\
\hline 1 & 0.1 & $2.488436 \times 10^{-1}$ & $2.476684 \times 10^{-1}$ & $2.476683 \times 10^{-1}$ & $2.476904 \times 10^{-1}$ & $2.477073 \times 10^{-1}$ \\
& 0.5 & $2.540209 \times 10^{-1}$ & $2.505365 \times 10^{-1}$ & $2.505363 \times 10^{-1}$ & $2.506012 \times 10^{-1}$ & $2.506486 \times 10^{-1}$ \\
& 0.9 & $2.591948 \times 10^{-1}$ & $2.578516 \times 10^{-1}$ & $2.578515 \times 10^{-1}$ & $2.578762 \times 10^{-1}$ & $2.578966 \times 10^{-1}$ \\
10 & 0.1 & $2.268524 \times 10^{-1}$ & $2.256753 \times 10^{-1}$ & $2.256753 \times 10^{-1}$ & $2.257005 \times 10^{-1}$ & $2.257192 \times 10^{-1}$ \\
& 0.5 & $2.319948 \times 10^{-1}$ & $2.285207 \times 10^{-1}$ & $2.285204 \times 10^{-1}$ & $2.285947 \times 10^{-1}$ & $2.286462 \times 10^{-1}$ \\
& 0.9 & $2.371526 \times 10^{-1}$ & $2.358195 \times 10^{-1}$ & $2.358194 \times 10^{-1}$ & $2.358476 \times 10^{-1}$ & $2.358697 \times 10^{-1}$ \\
\hline
\end{tabular}

Table 31. The absolute errors at some values of $x$ for each of the numerical schemes.

\begin{tabular}{cccccc}
\hline$t$ & $x$ & NSFD1 & NSFD2 & EEFDM & FIEFDM \\
\hline 1 & 0.1 & $1.175228 \times 10^{-3}$ & $1.175311 \times 10^{-3}$ & $1.153181 \times 10^{-3}$ & $1.136264 \times 10^{-3}$ \\
& 0.5 & $3.484373 \times 10^{-3}$ & $3.484620 \times 10^{-3}$ & $3.419683 \times 10^{-3}$ & $3.372316 \times 10^{-3}$ \\
& 0.9 & $1.343119 \times 10^{-3}$ & $1.343214 \times 10^{-3}$ & $1.318592 \times 10^{-3}$ & $1.298113 \times 10^{-3}$ \\
10 & 0.1 & $1.177087 \times 10^{-3}$ & $1.177163 \times 10^{-3}$ & $1.151921 \times 10^{-3}$ & $1.133274 \times 10^{-3}$ \\
& 0.5 & $3.474168 \times 10^{-3}$ & $3.474395 \times 10^{-3}$ & $3.400138 \times 10^{-3}$ & $3.348644 \times 10^{-3}$ \\
& 0.9 & $1.333150 \times 10^{-3}$ & $1.333237 \times 10^{-3}$ & $1.304959 \times 10^{-3}$ & $1.282913 \times 10^{-3}$ \\
\hline
\end{tabular}

Table 32. The relative errors at some values of $x$ for each of the numerical schemes.

\begin{tabular}{cccccc}
\hline$t$ & $x$ & NSFD1 & NSFD2 & EEFDM & FIEFDM \\
\hline 1 & 0.1 & $4.722757 \times 10^{-3}$ & $4.723090 \times 10^{-3}$ & $4.634160 \times 10^{-3}$ & $4.566176 \times 10^{-3}$ \\
& 0.5 & $1.371688 \times 10^{-2}$ & $1.371785 \times 10^{-2}$ & $1.346221 \times 10^{-2}$ & $1.327574 \times 10^{-2}$ \\
& 0.9 & $5.181890 \times 10^{-3}$ & $5.182259 \times 10^{-3}$ & $5.087262 \times 10^{-3}$ & $5.008252 \times 10^{-3}$ \\
10 & 0.1 & $5.188777 \times 10^{-3}$ & $5.189114 \times 10^{-3}$ & $5.077841 \times 10^{-3}$ & $4.995642 \times 10^{-3}$ \\
& 0.5 & $1.497520 \times 10^{-2}$ & $1.497617 \times 10^{-2}$ & $1.465609 \times 10^{-2}$ & $1.443413 \times 10^{-2}$ \\
& 0.9 & $5.621485 \times 10^{-3}$ & $5.621854 \times 10^{-3}$ & $5.502612 \times 10^{-3}$ & $5.409653 \times 10^{-3}$ \\
\hline
\end{tabular}


Table 33. $L_{1}$ and $L_{\infty}$ error norms with CPU times for the four numerical methods.

\begin{tabular}{ccccc}
\hline$t$ & Schemes & $L_{\mathbf{1}}$ Error & $\boldsymbol{L}_{\infty}$ Error & CPU Time $(\mathrm{Sec})$ \\
\hline 1 & NSFD1 & $2.302319 \times 10^{-3}$ & $3.484373 \times 10^{-3}$ & 0.0666 \\
& NSFD2 & $2.302482 \times 10^{-3}$ & $3.484620 \times 10^{-3}$ & 0.0669 \\
& EEFDM & $2.259624 \times 10^{-3}$ & $3.419683 \times 10^{-3}$ & 0.0658 \\
& FIEFDM & $2.227222 \times 10^{-3}$ & $3.372316 \times 10^{-3}$ & 0.0692 \\
10 & NSFD1 & $2.295375 \times 10^{-3}$ & $3.474168 \times 10^{-3}$ & 0.2304 \\
& NSFD2 & $2.295525 \times 10^{-3}$ & $3.474395 \times 10^{-3}$ & 0.2239 \\
& EEFDM & $2.246500 \times 10^{-3}$ & $3.400138 \times 10^{-3}$ & 0.2391 \\
& FIEFDM & $2.211288 \times 10^{-3}$ & $3.348644 \times 10^{-3}$ & 0.2418 \\
\hline
\end{tabular}

Case 9: $\alpha=1.0(\alpha=\beta), \beta=1.0$ and $\gamma=0.001$

Table 34. Absolute errors from four constructed schemes with the results of $[13,14]$ for $\alpha=1.0, \beta=1.0$, $\gamma=0.001$.

\begin{tabular}{|c|c|c|c|c|c|c|c|}
\hline$t$ & $x$ & NSFD1 & NSFD2 & EEFDM & FIEFDM & ADM & VIM \\
\hline \multirow[t]{3}{*}{0.05} & 0.1 & $8.13470 \times 10^{-9}$ & $8.13470 \times 10^{-9}$ & $7.80710 \times 10^{-9}$ & $7.55427 \times 10^{-9}$ & $1.87406 \times 10^{-8}$ & $1.87405 \times 10^{-8}$ \\
\hline & 0.5 & & $1.78494 \times$ & 1.7 & & 87406 & $1.87405 \times 10^{-8}$ \\
\hline & 0.9 & & $8.13526 \times 10$ & $7.80764 \times$ & $5 \times 10^{-9}$ & $1.87406>$ & $1.87405 \times 10^{-8}$ \\
\hline \multirow[t]{3}{*}{0.1} & 0.1 & $1.19758 \times$ & $1.19758 \times 10^{-8}$ & $1.13858 \times 10^{-8}$ & $1.11183 \times 10^{-8}$ & $3.74812 \times 10^{-8}$ & $3.74813 \times 10^{-8}$ \\
\hline & 0.5 & $3.02147 \times 1$ & $3.02147 \times 10^{-8}$ & $2.91176 \times 10^{-8}$ & $2.82588 \times 10^{-8}$ & $3.74813 \times 10^{-8}$ & $1.37481 \times 10^{-8}$ \\
\hline & 0.9 & $1.19770 \times 10^{-8}$ & $1.19770 \times 10^{-8}$ & $1.13870 \times 10^{-8}$ & $1.11195 \times 10^{-8}$ & $3.74813 \times 10^{-7}$ & $3.74813 \times 10^{-8}$ \\
\hline \multirow[t]{3}{*}{1.0} & 0.1 & $1.86367 \times 10^{-8}$ & $1.86366 \times 10^{-8}$ & $1.68648 \times 10^{-8}$ & $1.68635 \times 10^{-8}$ & $3.74812 \times 10^{-7}$ & $3.74812 \times 10^{-7}$ \\
\hline & 0.5 & $5.17712 \times 10^{-8}$ & $5.17712 \times 10^{-8}$ & $4.68494 \times 10^{-8}$ & $4.68457 \times 10^{-8}$ & $3.74812 \times 10^{-7}$ & $3.74813 \times 10^{-7}$ \\
\hline & 0.9 & $1.86393 \times 10^{-8}$ & $1.86392 \times 10^{-8}$ & $1.68670 \times 10^{-8}$ & $1.68656 \times 10^{-8}$ & $3.74812 \times 10^{-7}$ & $3.74813 \times 10^{-7}$ \\
\hline
\end{tabular}

In cases 10 and 11, we test the effectiveness of the fully implicit exponential finite difference scheme (FIEFDM) by taking a less refined time step $k=0.1$ for computations.

Case 10: $\alpha=\beta=0.5(\beta=\alpha), \gamma=0.001$ and $k=0.1$

Table 35. A comparison between the exact and numerical solutions with absolute and relative errors at some values of $x$.

\begin{tabular}{cccccc}
\hline$t$ & $x$ & Exact & FIEFDM & Absolute Error & Relative Error \\
\hline 1 & 0.1 & $5.000859 \times 10^{-4}$ & $5.000769 \times 10^{-4}$ & $9.040023 \times 10^{-9}$ & $1.807694 \times 10^{-5}$ \\
& 0.5 & $5.001249 \times 10^{-4}$ & $5.000998 \times 10^{-4}$ & $2.510788 \times 10^{-8}$ & $5.020322 \times 10^{-5}$ \\
& 0.9 & $5.001640 \times 10^{-4}$ & $5.001549 \times 10^{-4}$ & $9.040517 \times 10^{-9}$ & $1.807511 \times 10^{-5}$ \\
10 & 0.1 & $5.007711 \times 10^{-4}$ & $5.007621 \times 10^{-4}$ & $9.048705 \times 10^{-9}$ & $1.806954 \times 10^{-5}$ \\
& 0.5 & $5.008102 \times 10^{-4}$ & $5.007851 \times 10^{-4}$ & $2.513599 \times 10^{-8}$ & $5.019064 \times 10^{-5}$ \\
& 0.9 & $5.008492 \times 10^{-4}$ & $5.008402 \times 10^{-4}$ & $9.049199 \times 10^{-9}$ & $1.806771 \times 10^{-5}$ \\
\hline
\end{tabular}

Table 36. $L_{1}$ and $L_{\infty}$ error norms with CPU times for the FIEFDM.

\begin{tabular}{cccc}
\hline $\boldsymbol{t}$ & $\boldsymbol{L}_{\mathbf{1}}$ Error & $\boldsymbol{L}_{\infty}$ Error & CPU Time (Sec) \\
\hline 1.0 & $1.6571995 \times 10^{-8}$ & $2.5107882 \times 10^{-8}$ & 0.0331 \\
10.0 & $1.6589738 \times 10^{-8}$ & $2.5135986 \times 10^{-8}$ & 0.2818 \\
\hline
\end{tabular}


Case 11: $\alpha=0.5, \beta=10.0(\beta>>\alpha), \gamma=0.5$ and $k=0.1$

Table 37. A comparison between the exact and numerical solutions with absolute and relative errors at some values of $x$.

\begin{tabular}{cccccc}
\hline $\boldsymbol{t}$ & $\boldsymbol{x}$ & Exact & FIEFDM & Absolute Error & Relative Error \\
\hline 1 & 0.1 & $4.826732 \times 10^{-1}$ & $4.738086 \times 10^{-1}$ & $8.864652 \times 10^{-3}$ & $1.836574 \times 10^{-2}$ \\
& 0.5 & $4.885113 \times 10^{-1}$ & $4.627574 \times 10^{-1}$ & $2.575394 \times 10^{-2}$ & $5.271924 \times 10^{-2}$ \\
& 0.9 & $4.924132 \times 10^{-1}$ & $4.841520 \times 10^{-1}$ & $8.261271 \times 10^{-3}$ & $1.677711 \times 10^{-2}$ \\
10 & 0.1 & $5.000000 \times 10^{-1}$ & $5.000000 \times 10^{-1}$ & $2.831070 \times 10^{-15}$ & $5.662141 \times 10^{-15}$ \\
& 0.5 & $5.000000 \times 10^{-1}$ & $5.000000 \times 10^{-1}$ & $8.493211 \times 10^{-15}$ & $1.698641 \times 10^{-14}$ \\
& 0.9 & $5.000000 \times 10^{-1}$ & $5.000000 \times 10^{-1}$ & $2.720052 \times 10^{-15}$ & $5.440091 \times 10^{-15}$ \\
\hline
\end{tabular}

Table 38. $L_{1}$ and $L_{\infty}$ error norms with CPU times for the FIEFDM.

\begin{tabular}{cccc}
\hline $\boldsymbol{t}$ & $\boldsymbol{L}_{\mathbf{1}}$ Error & $\boldsymbol{L}_{\boldsymbol{\infty}}$ Error & CPU Time $(\mathrm{Sec})$ \\
\hline 1.0 & $1.659845 \times 10^{-2}$ & $2.575394 \times 10^{-2}$ & 0.0256 \\
10.0 & $5.462297 \times 10^{-15}$ & $8.493206 \times 10^{-15}$ & 0.3112 \\
\hline
\end{tabular}

We next present plots of the initial, exact and numerical solutions for the eleven cases in Figures 10-29.

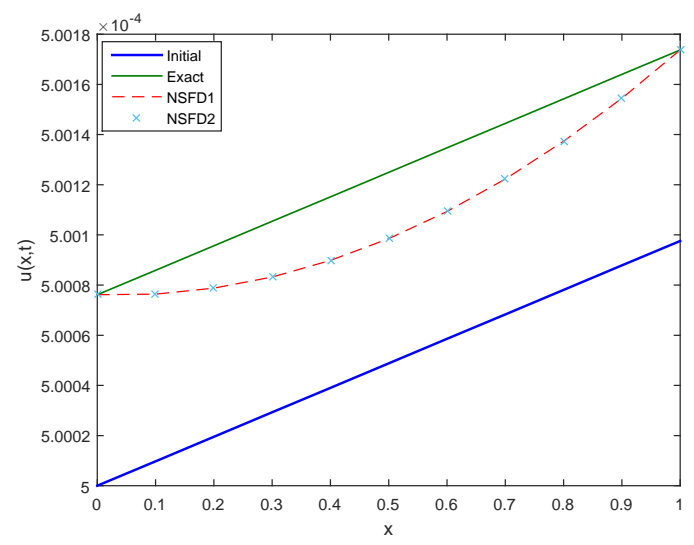

Figure 10. Comparison between Initial, Exact, NSFD1 and NSFD2 profiles for Case 1 at $t=1.0$.

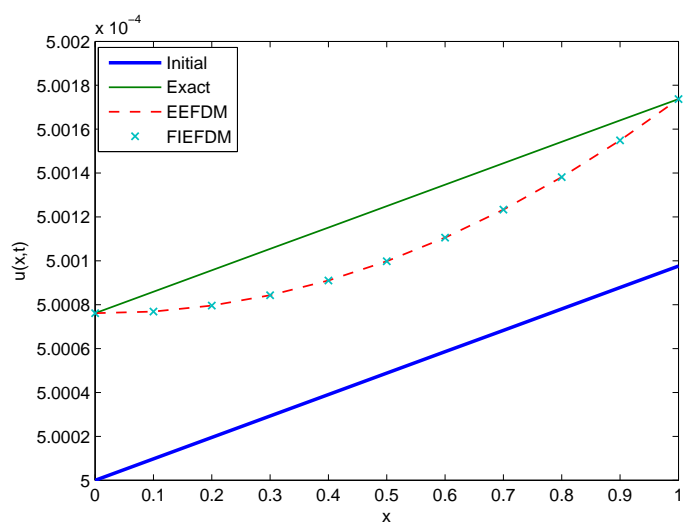

Figure 11. Comparison between Initial, Exact, EEFDM and FIEFDM profiles for Case 1 at $t=1.0$. 


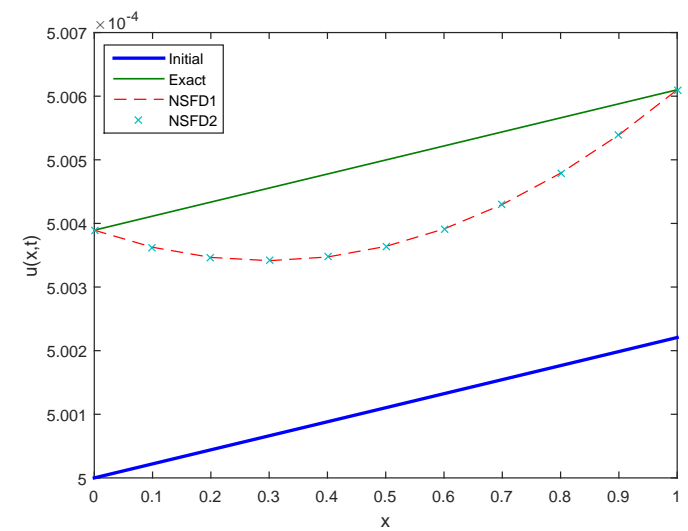

Figure 12. Comparison between Initial, Exact, NSFD1 and NSFD2 profiles for Case 2 at $t=1.0$.

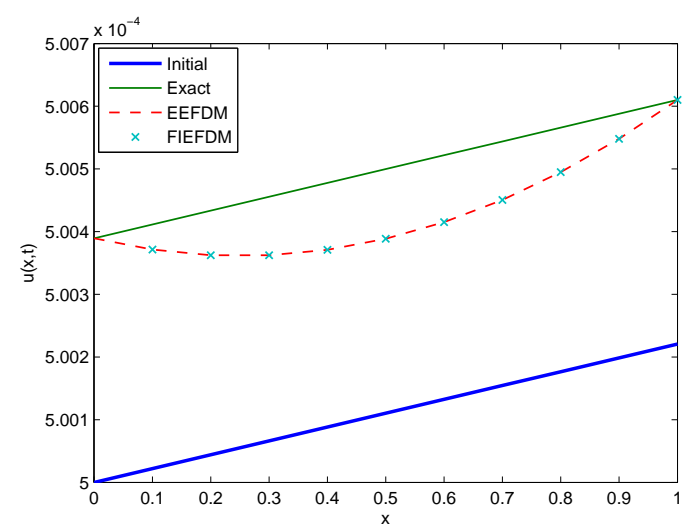

Figure 13. Comparison between Initial, Exact, EEFDM and FIEFDM profiles for Case 2 at $t=1.0$.

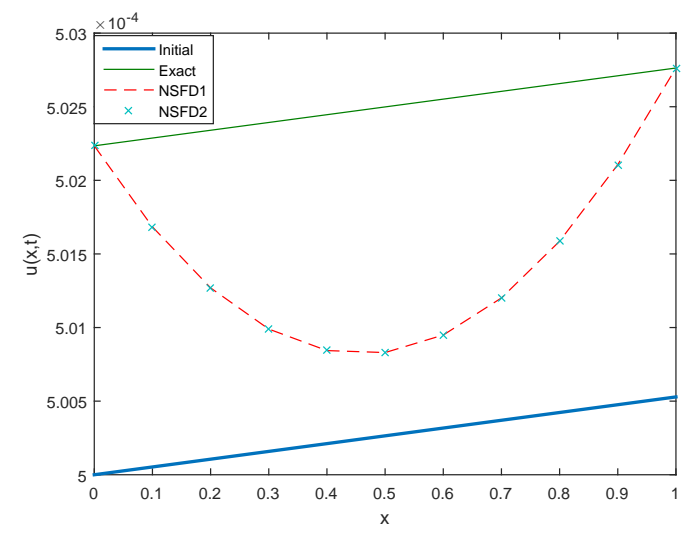

Figure 14. Comparison between Initial, Exact, NSFD1 and NSFD2 profiles for Case 3 at $t=1.0$. 


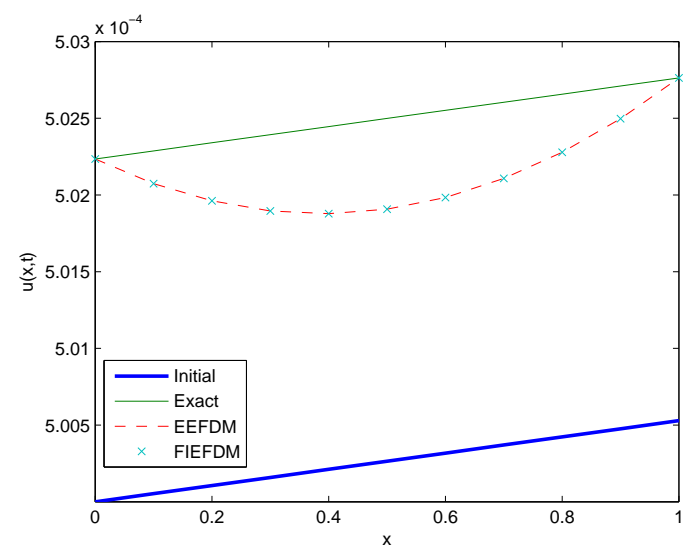

Figure 15. Comparison between Initial, Exact, EEFDM and FIEFDM profiles for Case 3 at $t=1.0$.

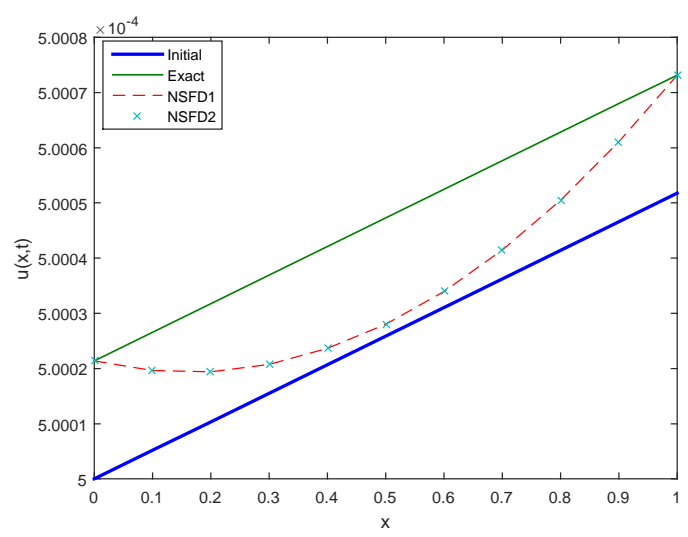

Figure 16. Comparison between Initial, Exact, NSFD1 and NSFD2 profiles for Case 4 at $t=1.0$.

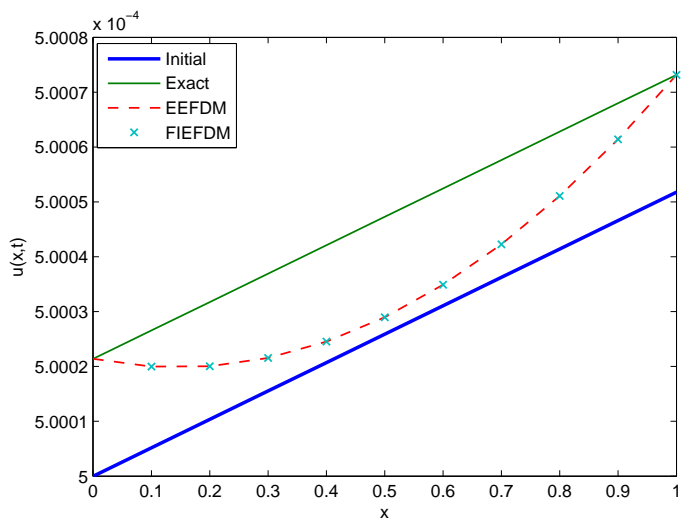

Figure 17. Comparison between Initial, Exact, EEFDM and FIEFDM profiles for Case 4 at $t=1.0$. 


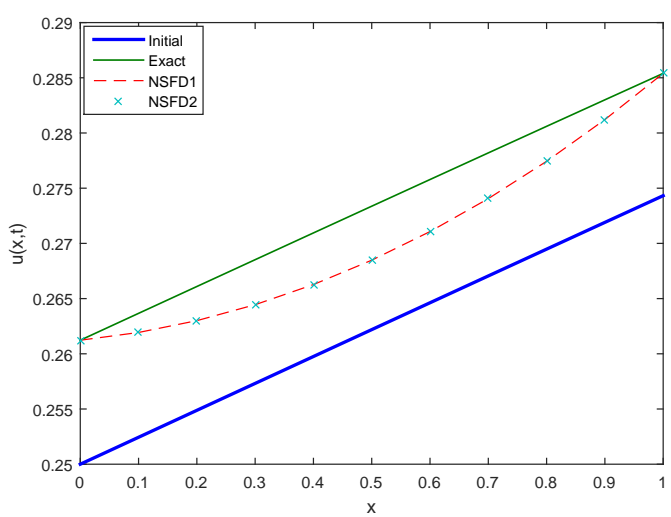

Figure 18. Comparison between Initial, Exact, NSFD1 and NSFD2 profiles for Case 5 at $t=1.0$.

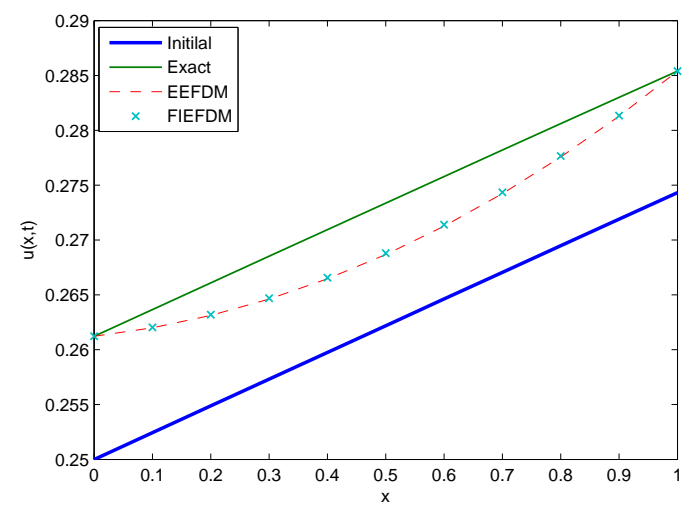

Figure 19. Comparison between Initial, Exact, EEFDM and FIEFDM profiles for Case 5 at $t=1.0$.

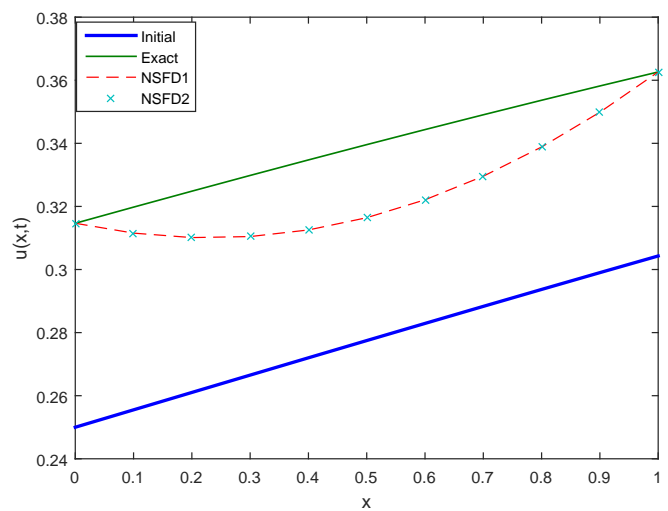

Figure 20. Comparison between Initial, Exact, NSFD1 and NSFD2 profiles for Case 6 at $t=1.0$. 


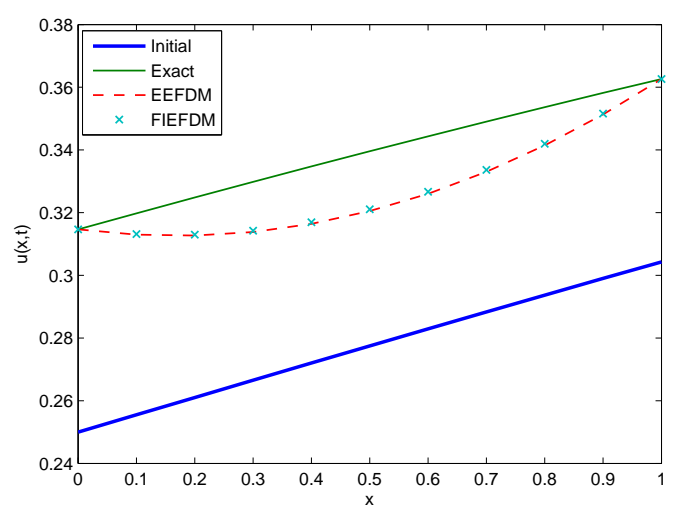

Figure 21. Comparison between Initial, Exact, EEFDM and FIEFDM profiles for Case 6 at $t=1.0$.

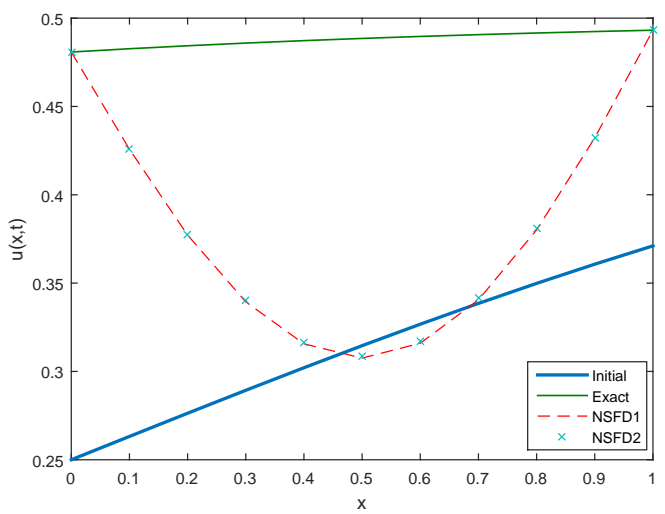

Figure 22. Comparison between Initial, Exact, NSFD1 and NSFD2 profiles for Case 7 at $t=1.0$.

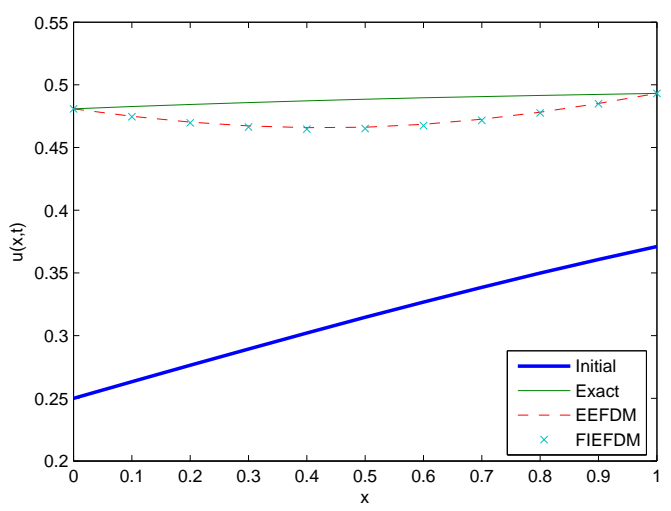

Figure 23. Comparison between Initial, Exact, EEFDM and FIEFDM profiles for Case 7 at $t=1.0$. 


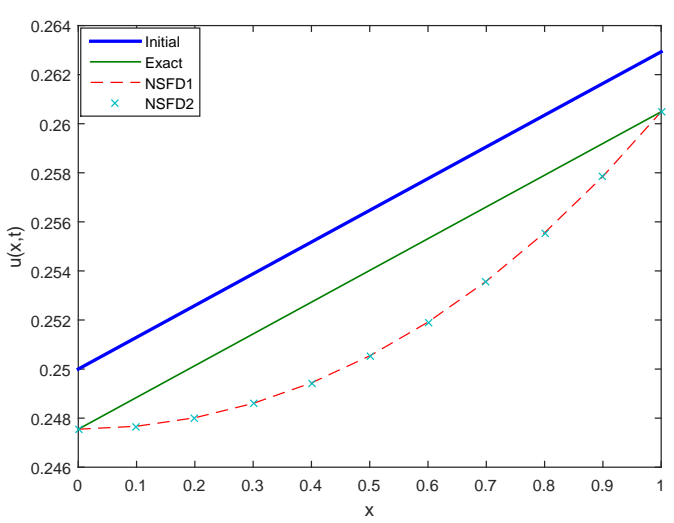

Figure 24. Comparison between Initial, Exact, NSFD1 and NSFD2 profiles for Case 8 at $t=1.0$.

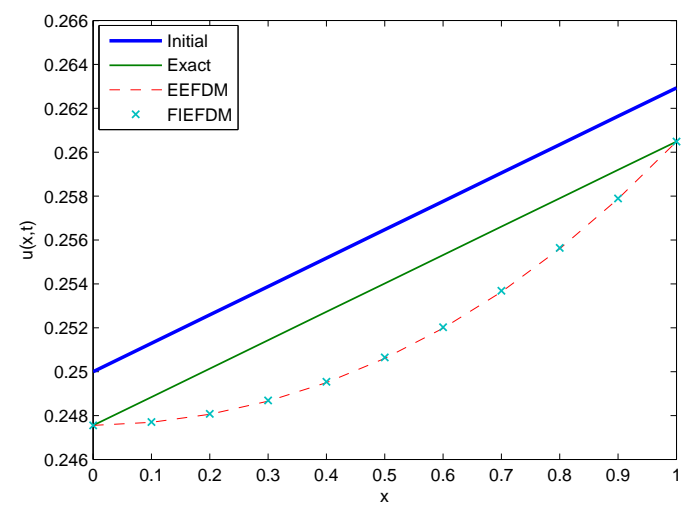

Figure 25. Comparison between Initial, Exact, EEFDM and FIEFDM profiles for Case 8 at $t=1.0$.

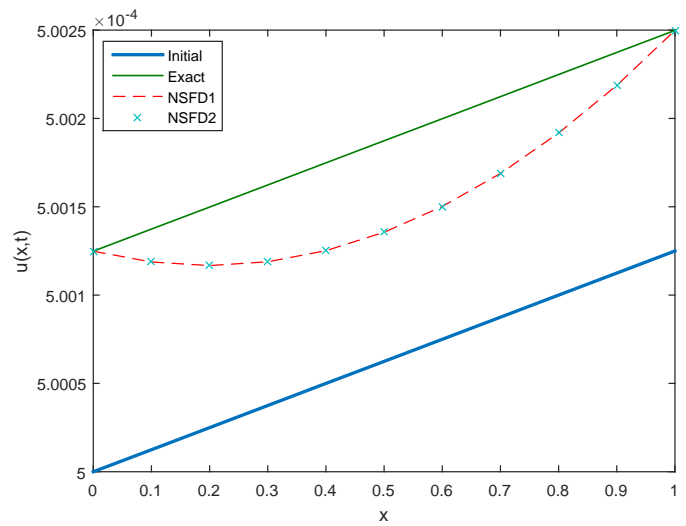

Figure 26. Comparison between Initial, Exact, NSFD1 and NSFD2 profiles for Case 9 at $t=1.0$. 


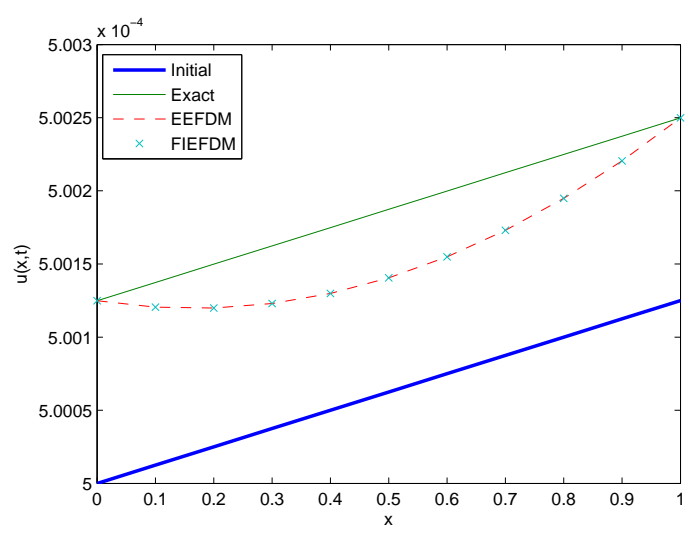

Figure 27. Comparison between Initial, Exact, EEFDM and FIEFDM profiles for Case 9 at $t=1.0$.

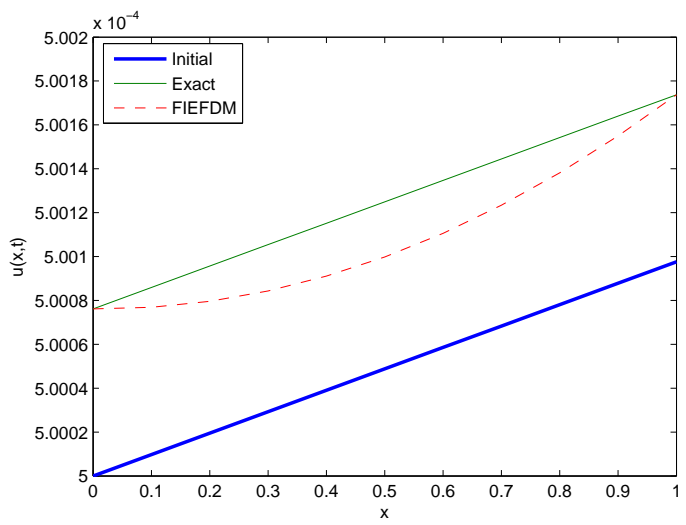

Figure 28. Comparison between Initial, Exact and FIEFDM profiles for Case 10 at $t=1.0$.

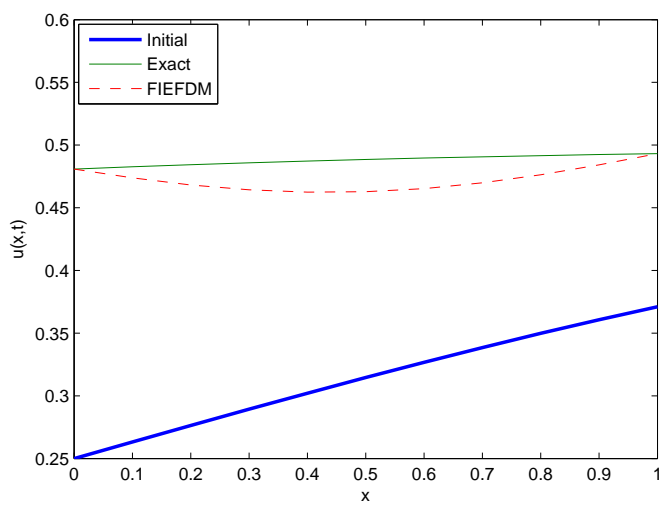

Figure 29. Comparison between Initial, Exact and FIEFDM profiles for Case 11 at $t=1.0$.

\section{Conclusions}

In this paper, we have obtained numerical solutions to the Burgers-Huxley equation with specified initial and boundary conditions using two novel non-standard finite difference and two exponential finite difference schemes. These types of schemes are very recent for such partial differential equations. The positivity condition of the two NSFD schemes are dependent on $k, \beta$ and $h$. We considered eleven test cases, which made use of two values of $\gamma=0.001$ and 0.5. The last two test cases are for FIEFDM only, where we used a larger time step size.

For $\gamma=0.001$, we observe that, for the three different regimes $(\alpha=\beta, \alpha<\beta, \alpha>\beta$, ), all the four schemes are very efficient. The relative error is of order $10^{-5}$. The CPU time for the FIEFDM is slightly larger than the other three explicit schemes. For $\gamma=0.5$, we observe that, for all the three different 
regimes, the four schemes perform quite well and the relative error is of order $10^{-2}, 10^{-3}$ and $10^{-4}$. We also compared our four methods with adomian decomposition and variational iteration method for the case $\alpha=\beta=1.0, \gamma=0.001$ and our methods slightly performed better.

In reference to Figures 14 and 15, we can observe that EEFDM amd FIEFDM are much better than NSFD1 and NSFD2 for the case $\alpha=0.5, \beta=10.0, \gamma=0.001$ at time $t=1.0$.

If we refer to Figures 22 and 23, we can observe that EEFDM and FIEFDM are much better than NSFD1, NSFD2 for the case $\alpha=0.5, \beta=10.0, \gamma=0.5$ at time $t=1.0$.

There is not much difference in performance of FIEFDM at $k=0.1$ as compared to $k=0.005$, hence a larger time step size can be used.

We would like to extend this study to solve generalised Burgers-Huxley equation using a more challenging numerical experiment, possibly one which consists of a shock-like profile.

Author Contributions: These authors contributed equally to this work.

Funding: A. R. Appadu is grateful to Nelson Mandela University for seed funding which enabled him to present his work at ICNAAM 2019. Yusuf O. Tijani is grateful to Nelson Mandela University for providing some funding to cover first year registration fees at the university.

Acknowledgments: The authors would like to thank the three anonymous reviewers for the insightful comments and suggestions, which have enabled the authors to considerably improve the paper.

Conflicts of Interest: The authors declare no conflict of interest.

\section{Abbreviations}

The following abbreviations are used in this manuscript:

NSFD Nonstandard Finite Difference

EEFDM Explicit Exponential Finite Difference Method

FIEFDM Fully Implicit Exponential Finite Difference Method

MATLAB Matrix Laboratory

\section{References}

1. Albowitz, M.J.; Clarkson, P.A. Solitons, Nonlinear Evolution Equations and Inverse Scattering Transform; University Press: Cambridge, UK, 1990.

2. Lawrence, C.E. Partial Differential Equation; Graduate Studies in Mathematics; American Mathematical Society: Berkeley, CA, USA, 1997; pp. 1-662.

3. Estevez, P.G. Non-classical symmetries and the singular manifold method: The Burgers and the Burgers-Huxley equations. J. Phys. A Math. Gen. 1994, 27, 2113-2127. [CrossRef]

4. Estevez, P.G.; Gordoa, P.R. Nonclassical symmetries and the singular manifold method: Theory and six examples. Stud. Appl. Math. 1995, 95, 73-113. [CrossRef]

5. Chou, M.H.; Lin, Y.T. Exotic dynamic behavior of the forced FitzHugh-Nagumo equations. Comput. Math. Appl. 1996, 32, 109-124. [CrossRef]

6. Olver, P. Applications of Lie Groups to Differential Equations, Graduate Texts in Mathematics; Springer: New York, NY, USA, 1994; Volume 107.

7. Zhao, X.; Tang, D.; Wang, L. New soliton-like solutions for KdV equation with variable coefficient. Phys. Lett. A 2005, 346, 228-291. [CrossRef]

8. Yomba, E. The extended Fan's sub-equation method and its applications to KdV-MkdV, BKK and variant Boussinesq equations. Phys. Lett. A 2005, 336, 463-476. [CrossRef]

9. Wazwaz, A.M. Solutions and periodic solutions for the fifth-order KdV equation. Appl. Math. Lett. 2006, 19, 1162-1167. [CrossRef]

10. Wang, M. Exact solutions for a compound KdV-Burgers equation. Phys. Lett. A 1996, 213, 79-287. [CrossRef]

11. Biswas, A. Anjan S1-soliton solution of the $\mathrm{K}(\mathrm{m}, \mathrm{n})$ equation with generalized evolution. Phys. Lett. A 2008, 25, 4601-4602. [CrossRef]

12. Yu, X.; Gao, Y.T.; Sun, Z.Y.; Liu, Y. N-soliton solutions, Backlund transformation and Lax pair for a generalized variable-coefficient fifth-order Korteweg-de Vries equation. Phys. Scr. 2010, 81, 045402. [CrossRef] 
13. Ismail, H.N.A.; Raslan, K.; Abd Rabboh, A.A. Adomian decomposition method for burgers-Huxley and burgers-Fisher equations. Appl. Math. Comput. 2004, 1, 291-301.

14. Batiha, M.S.M.; Noorani, B.; Hashim, I. Application of variational iteration method to the generalized burgers-huxley equation. Chaos Solitons Fractals 2008, 36, 660-663. [CrossRef]

15. Biazar, J.; Mohammadi, F. Application of differential transform method to the generalized burgers-huxley equation. Appl. Appl. Math. 2010, 5, 1726-1750.

16. Yaghouti, M.R.; Zabihi, A. Application of Laplace decomposition method for burgers-huxley and burgers-fisher equations. J. Math. Model. 2013, 1, 41-67.

17. Molabahramia, A.; Khan, F. The homotopy analysis method to solve the burgers-huxley equation. Nonlinear Anal. Real World Appl. 2009, 10, 589-600. [CrossRef]

18. Mittal, R.C.; Jiwari, R. A numerical study of burger-huxley equation by differential quadrature method. J. Appl. Math. Mech. 2009, 5, 1-9.

19. Javidi, M. A numerical solution of the generalized burgers-huxley equation by spectral collocation method. Appl. Math. Comput. 2006, 178, 338-334.

20. Tomasiello, S. Numerical solutions of the burgers-huxley equation by the IDQ method. Int. J. Comput. Math. 2010, 87, 129-140. [CrossRef]

21. Ray, S.S.; Gupta, A. Comparative analysis of variational iteration method and Haar wavelet method for the numerical solutions of Burgers-Huxley and Huxley equations. J. Math. Chem. 2014, 52, 1060-1080.

22. Ray, S.S.; Gupta, A. Application of novel schemes based on Haar wavelet collocation method for numerical simulations of Burger and Boussinesq-Burger equations. Appl. Math. Inf. Sci. 2016, 10, 1513-1524. [CrossRef]

23. Chen, Z.; Gumel, A.B.; Micken, R.E. Nonstandard discretizations of the generalized nagumo reaction-diffusion equation. Numer. Methods Partial Differ. Equ. 2003, 19, 363-379. [CrossRef]

24. Kyrychko, Y.N.; Bartuccelli, M.; Blysuss, K.B. Persistence of travelling wave solutions of a fourth order diffusion system. J. Comput. Appl. Math. 2005, 176, 433-443. [CrossRef]

25. Anguelov, R.; Lubuma, J.M.S. Contributions to the Mathematics of the Nonstandard Finite Difference Method and Applications. Numer. Methods Partial Differ. Equ. 2001, 17, 518-543. [CrossRef]

26. Mickens, R.E. Application of Nonstandard Finite Difference Scheme; World Scientific: Singapore, 2000; pp. 1-261.

27. Mickens, R.E.; Gumel, A.B. Construction and analysis of a non-standard finite difference scheme for the Burgers-Fisher equation. J. Sound Vib. 2002, 4, 791-797. [CrossRef]

28. Zhang, L.; Wang, L.; Xiaohu, D. Exact finite difference scheme and nonstandard finite difference scheme for burgers and burgers-fisher equations. J. Appl. Math. 2014, 597926. [CrossRef]

29. Agbavon, K.M.; Appadu, A.R. Construction and analysis of some nonstandard finite difference methods for the Fitzhugh-Nagumo equation. Numer. Methods Partial Differ. Equ. In Review.

30. Appadu, A.R.; Lubuma, J.M.S.; Mphephu, N. Computational study of three numerical methods for some linear and nonlinear advection-diffusion-reaction problems. Prog. Comput. Fluid Dyn. 2017, 17, 114-129. [CrossRef]

31. Appadu, A.R. Numerical solution of the $1 \mathrm{D}$ advection-diffusion equation using standard and nonstandard finite difference schemes. J. Appl. Math. 2013, 2013, 734374. [CrossRef]

32. Agabvon, K.M.; Appadu, A.R.; Khumalo, M. On the numerical solution of fishers equation with coefficient of diffusion term much smaller than coefficient of reaction term. Adv. Differ. Equ. 2019, 146, 33. [CrossRef]

33. Chapwanya, M.; Appadu, A.R.; Jejeniwa, M.O.; Lubuma, J.M.S. An explicit nonstandard finite difference scheme for the Fitzhugh-Nagumo equations. Int. J. Comput. Math. 2019, 96, 1993-2009. [CrossRef]

34. Mickens, R.E. Nonstandard finite difference schemes for reaction-diffusion equations. Numer. Methods Partial Differ. Equ. 1999, 15, 201-215. [CrossRef]

35. Jordan, P.M. A nonstandard finite difference scheme for nonlinear heat transfer in a thin finite rod. J. Differ. Equ. Appl. 2003, 9, 1015-1021. [CrossRef]

36. Aderogba, A.A.; Chapwanya, M. Positive and bounded nonstandard finite difference scheme for the hodgkin-huxley equations. Jpn. J. Ind. Appl. Math. 2018, 35, 773-785. [CrossRef]

37. Zibaei, M.; Zeinadini, S.; Namjoo, M. Numerical solutions of Burgers-Huxley equation by exact finite difference and NSFD schemes. J. Differ. Equ. Appl. 2016, 22, 1098-1113. [CrossRef]

38. Oluwaseye, A.; Talitha, W. Nonstandard finite difference scheme for a Tacoma Narrows Bridge model. Appl. Math. Model. 2018, 62, 223-236. 
39. Dai, W. Nonstandard finite difference schemes for solving nonlinear micro heat transport equations in double-layered metal thin films exposed to ultrashort pulsed lasers. In Advances in the Applications of Nonstandard Finite Difference Schemes; Mickens, R.E., Ed.; World Scientific: Hackensack, NJ, USA, 2005; pp. 191-248.

40. Díaz-Rodríguez, M.; González-Parra, G.; Arenas, A.J. Nonstandard numerical schemes for modeling a 2-DOF serial robot with rotational spring-damper-actuators. Int. J. Numer. Methods Biomed. Eng. 2011, 27, 1211-1224. [CrossRef]

41. Inan, B.; Bahadir, A.R. A numerical solution of the Burgers equation using a Crank-Nicolson exponential finite difference method. J. Math. Comput. Sci. 2014, 4, 849-860.

42. Macías-Díaz, J.E. A modified exponential method that preserves structural properties of the solutions of the Burgers-Huxley equation. Int. J. Comput. Math. 2017. [CrossRef]

43. Bhattacharya, M.C. An explicit conditionally stable finite difference equation for heat conduction problems. Int. J. Numer. Method Eng. 1985, 21, 239-265. [CrossRef]

44. Inan, B.; Bahadir, A.R. Numerical solution of the one-dimensional burgers equation: Implicit and fully implicit exponential finite difference methods. Pramana J. Phys. 2018, 81, 547-556. [CrossRef]

45. Bahadir, A.R. Exponential finite-difference method applied to korteweg-de vries equation for small times. Appl. Math. Comput. 2005, 160, 675-682.

46. İnan, B. Finite difference methods for the generalized huxley and burgers-huxley equations. Kuwait J. Sci. 2017, 44, 20-27.

47. İnan, B. An exponential finite difference method based on Padé approximation. Celal Bayar Univ. Sci. 2017, 13, 71-80. [CrossRef]

48. Macías-Díaz, J.E.; İnan, B. Numerical efficiency of some exponential methods for an advection-diffusion equation. Int. J. Comput. Math. 2019, 96, 1005-1029. [CrossRef]

49. Appadu, A.R.; İnan, B.; Tijani, Y.O. Comparison of some numerical methods for the Burgers-Huxley equation. To appear in AIP Conference Proceedings.

50. Griffiths, W.G.; Schiesser, E.W. Traveling Wave Analysis of Partial Differential Equation: Numerical and Analytical Methods with MATLAB and Maple; Academic Press: Cambridge, MA, USA, 2011; p. 461. ISBN: 9780123846525.

51. Deng, X. Travelling wave solutions for the generalized Burgers-Huxley equation. Appl. Math. Comput. 2008, 204, 733-737. [CrossRef]

52. Burgers, J.M. A mathematical model illustrating the theory of turbulence. In Advances in Applied Mechanics; Academic Press: New York, NY, USA, 1948; Volume 1, pp. 171-199.

53. Nourazar, S.S.; Soori, M.; Nazari-Golshan, A. On the Exact Solution of Newell-Whitehead-Segel Equation Using the Homotopy Perturbation Method. Aust. J. Basic Appl. Sci. 2011, 5, 1400-1411.

54. Wang, Z.S.; Zhuo, X.Y.; Lu, Y.K. Solitary wave solutions of the generalised Burgers-Huxley equation. J. Phys. A Math. Gen. 1990, 23, 271-274. [CrossRef]

55. Mickens, R.E. Pitfalls in the numerical integration of differential equations. In Analytical Techniques for Material Characterization; Collins, W.E., Chowdari, B.V.R., Radhakrishna, S., Eds.; World Scientific: Singapore, 1987; Volume 17, pp. 123-143.

56. Mickens, R.E.; Smith, A. Finite-Difference Model of Ordinary Differential Equations: Influence of Denominator Function. J. Franklin Inst. 1990, 327, 143-149. [CrossRef]

57. Mickens, R.E. Lie methods in mathematical modeling: Difference equation models of differential equations. Math. Comput. Model. 1988, 11, 528-530. [CrossRef]

58. Hilderband, F.B. Finite-Difference Equations and Simulations; Prentice-Hall: Englewood Cliffs, NJ, USA, 1968; p. 338.

59. Taha, T.; Ablowitz, M. Analytical and numerical aspects of certain nonlinear evolution equations III. Numerical Korteweg-de Vries equation. J. Comput. Phys. 1984, 2, 231-235. [CrossRef]

(C) 2019 by the authors. Licensee MDPI, Basel, Switzerland. This article is an open access article distributed under the terms and conditions of the Creative Commons Attribution (CC BY) license (http:/ / creativecommons.org/licenses/by/4.0/). 